\title{
Neural computation underlying rapid learning and dynamic memory recall for sensori-motor control in insects.
}

\author{
Hannes Rapp ${ }^{\mathrm{a}}$, Martin Paul Nawrot ${ }^{\mathrm{a}, *}$ \\ ${ }^{a}$ Computational Systems Neuroscience, Institute of Zoology, University of Cologne, Zülpicher Straße 47b, Cologne, Germany
}

\begin{abstract}
Foraging is a vital behavioral task for living organisms. Behavioral strategies and abstract mathematical models thereof have been described in detail for various species. To explore the link between underlying nervous systems and abstract computational principles we present how a biologically detailed neural circuit model of the insect mushroom body implements sensory processing, learning and motor control. We focus on cast \& surge strategies employed by flying insects when foraging within turbulent odor plumes. Using a synaptic plasticity rule the model rapidly learns to associate individual olfactory sensory cues paired with food in a classical conditioning paradigm. Without retraining, the system dynamically recalls memories to detect relevant cues in complex sensory scenes. Accumulation of this sensory evidence on short timescales generates cast \& surge motor commands. Our systems approach is generic and predicts that population sparseness facilitates learning, while temporal sparseness is required for dynamic memory recall and precise behavioral control.

Keywords: mushroom body, sparse coding, sensori-motor control, foraging, olfaction, plasticity, transfer-learning, spiking neural network, Artificial Intelligence
\end{abstract}

\section{Introduction}

Navigating towards a food source during foraging requires dynamical sensory processing, accumulation of sensory evidence and appropriate high level motor control. Navigation based on an animals' olfactory sense is a challenging task due to the complex spatiotemporal landscape of odor molecules. A core aspect of foraging is the acquisition of sensory 5 cue samples in the natural environment where odor concentrations vary rapidly and steeply across space. Experimental access to the neural substrate is challenging in freely behaving insects. Biologically realistic models thus play a key role in investigating the relevant computational mechanisms. Consequently, recent efforts at understanding foraging behavior have focused on identifying viable computational strategies for making navigation decisions (Vergassola et al., 2007; Masson, 2013; Gaudry et al., 2012; Hein et al., 2016; Baddeley et al., 2009; Baker et al., 2018; Cardé and Willis, 2008).

An odor plume is often considered a volume wherein odor concentration is generally above some behavioral threshold. At macroscopic scales and in a natural environment, however, plumes are turbulent (Murlis et al., 1992; Crimaldi et al., 2002). In turbulent conditions a plume breaks up into complex and intermittent filamentous structures that are interspersed with clean air pockets or below behavioral threshold concentration patches (Celani, 2014; Kree et al., 2013; Connor et al., 2018). The dispersing filaments form the cone-like shape of the macroscopic plume where the origin of the cone yields the position of the odor source. When entering the cone, flying insects encounter odor filaments as discrete, short-lived sensory events in time.

\footnotetext{
${ }^{*}$ Corresponding author

Email address: martin.nawrot@uni-koeln.de (Martin Paul Nawrot)
} 
Several features have been derived from the statistics of an odor plume that provide information regarding the location of the odor source (Balkovsky and Shraiman, 2002; Celani, 2014; Crimaldi et al., 2002; Murlis et al., 2000; Shraiman and Siggia, 2000). The mean concentration varies smoothly in lateral and longitudinal directions of time-averaged (and laminar) plumes. However, for behavioral strategies animals cannot afford the time it takes to obtain stable macroscopic estimates of mean concentrations (Murlis et al., 1992). Vickers et al. (2001) and Park et al. (2016) proposed the time interval between odor encounters as an informative olfactory feature while Crimaldi et al. (2002) suggested intermittency, the probability of the odor concentration being above some behavioral threshold, as the relevant feature. However, similarly to estimating mean concentration, acquiring a sufficient number of samples for stable estimates of these quantities exceeds 25 the time typically used to form behavioral decisions (Murlis et al., 1992). Hence, obtaining time averaged quantities is not an optimal strategy to guide navigation decisions as concluded by Boie et al. (2018).

Most animals perform searches at large distances from the odor source where the intermittency of plumes becomes a more severe problem as available sensory cues become more sparse in space and time. Thus, strategies that exploit brief, localized sensory cues for navigation have been studied by several groups. One strategy for medium and long-range navigation that has consistently been observed across species of flying insects emerges from a sequence of chained sensorimotor reflexes: casting \& surging (van Breugel et al., 2015; Gaudry et al., 2012). Encountering a whiff of odor triggers an upwind surge behavior, during which the insect travels parallel to the wind direction. After losing track of the plume it evokes a crosswind cast behavior, in which a flight path perpendicular to the direction of air flow is executed. Performing repeated casts by U-turning allows the insect to reenter and locate the plume in order to trigger the next upwind surge (Cardé and Willis, 2008; van Breugel and Dickinson, 2014; van Breugel et al., 2015; Vickers and Baker, 1994; Riffell et al., 2014; Pang et al., 2018; Budick and Dickinson, 2006). As the subject approaches the source it increasingly makes use of visual cues for navigation as the plume narrows down. (van Breugel and Dickinson, 2014; Saxena et al., 2018).

A number of studies have proposed abstract mathematical models for optimal search algorithms that assumed different types of relevant navigational cues. The infotaxis method proposed in Vergassola et al. (2007) depends on extensive memory 40 and priors regarding a plume's structure. Contrary, in van Breugel and Dickinson (2014) only local cues are used. A standard algorithm for navigational problems in robotics is simultaneous localisation and mapping (SLAM), which has been used in Baddeley et al. (2009) to study olfactory navigation in bumblebees. An algorithm that works without space perception has been proposed by Masson (2013) using a standardized projection of the probability of source position and minimization of a free energy along the trajectory. Finally, the work of Pang et al. (2018) compares several models and shows that it is difficult to discriminate between different models based on behavioral responses. A recent work by Boie et al. (2018) using information-theoretic analysis shows that plumes contain both, spatial and temporal information about the source's position.

While all of these previous mathematical methods for olfactory search algorithms have proven to successfully solve this task based on the respective assumptions, they share the same major drawback: none of them uses the computational substrate of the brain, spiking neurons and networks thereof. Instead, all methods make heavy use of symbolic math and advanced mathematical concepts that are not available to the biological brain. It is further unclear how and to what extend these methods could be implemented or learned by the nervous system. Additionally, the problem of navigation and foraging is often considered as an isolated task, independent from sensory processing.

Our approach distills recent experimental results to formulate a biologically plausible and detailed spiking neural 
regarding the anatomy (e.g. (Aso et al., 2014a; Caron et al., 2013; Xu et al., 2020)) and neurophysiology (e.g. (Ito et al., 2008; Kazama and Wilson, 2009; Demmer and Kloppenburg, 2009; Szyszka et al., 2014; Inada et al., 2017; Egea-Weiss et al., 2018)) of insect olfaction and basic computational features (Litwin-Kumar et al., 2017; Kloppenburg and Nawrot, 2014; Betkiewicz et al., 2020). We follow the idea of compositionality, a widely used concept in mathematics, semantics and linguistics. According to this principle, the meaning of a complex expression is a function of the meanings of its constituent expressions (Frege principle (Hintikka, 1984)). In the present case of foraging and navigation this means dynamically recombining memories of individual (temporal and spatial) sensory cues present within a plume.

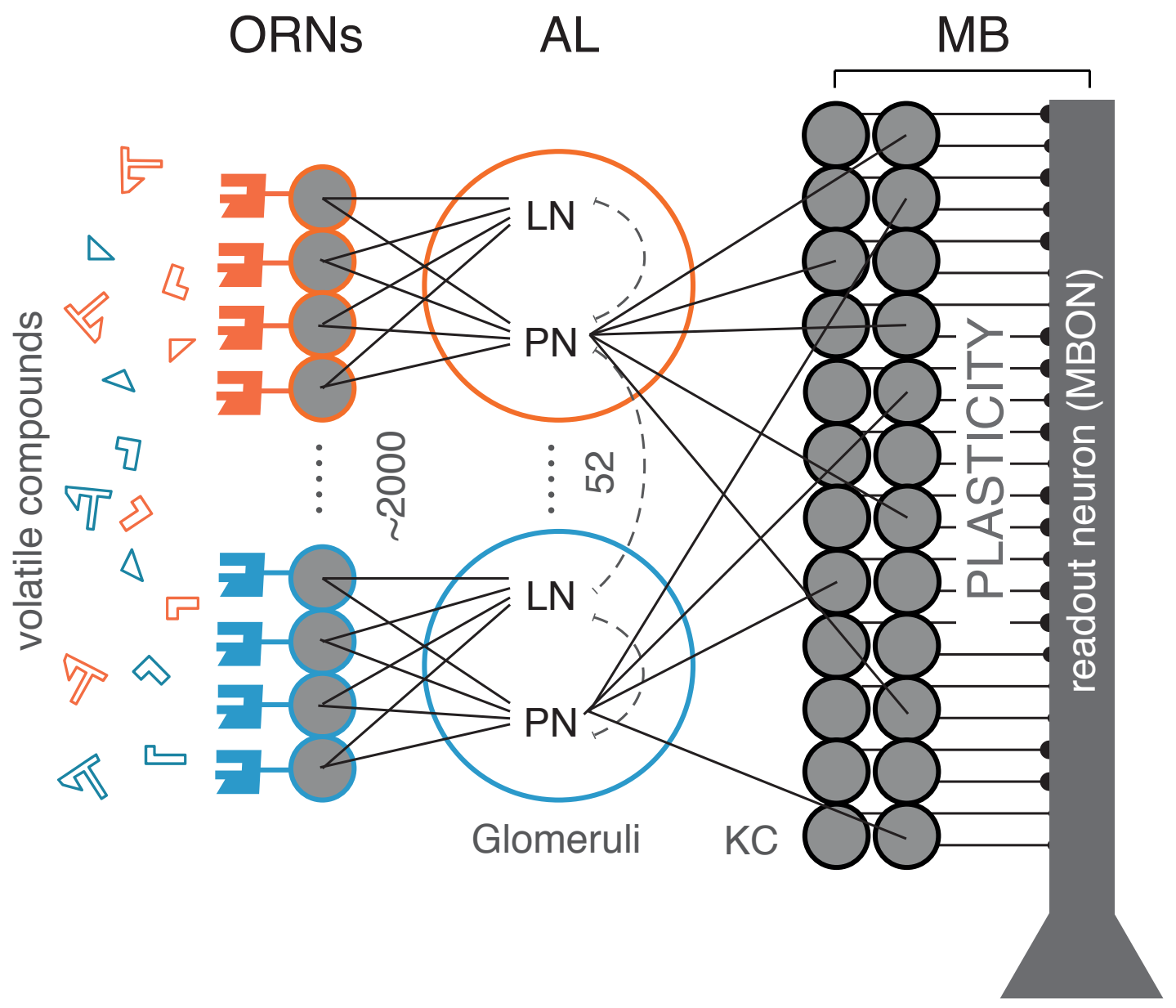

Figure 1: Spiking network model of the insect olfactory system. 2048 olfactory receptor neurons (ORNs) at the antennae bind and respond to volatile odorant compounds. ORNs expressing the same genetic phenotype (52 different receptor types) project to the same Glumerus in the antenal lobe (AL). Each of the 52 Glomeruli consitutes of one projection (PN) and local interneuron (LN). LNs form lateral inhibitory connections among Glomeruli and PNs randomly connect to a large population of Kenyon Cells (KC) where each KC receives input from 6 random PNs on average. Sensory processing, learning and memory is performed by the Mushroom body (MB) with Kenyon Cells $(\mathrm{KC})$ and a fully connected single, plastic readout neuron (mushroom body output neuron (MBON)). The overall architectural bauplan of the olfactory system is homologous across species. Here the specific numbers of neurons within each population and connectivity are taken from the connectome of the mushroom body of the adult Drosophila melanogaster

\section{Results}

We approach the problem of foraging by decomposition into four components: First, sensory processing with temporal 
individual odor identities. Third, the time-dependent detection of valenced cues resulting from encounters of discrete odor filaments to provide an ongoing and robust estimate of sensory cue evidence. Fourth, the translation into online motor command signals to drive appropriate behavior.

For sensory processing we use a three-layer spiking neural network model of the insect olfactory pathway (see Fig 1). The generic blueprint of the insect olfactory system is homologous across species and comprises three successive processing stages (see Methods for details): The periphery with olfactory receptor neurons (ORNs), the antennal lobe (AL) and the MB. Excitatory feed-forward connections across layers from ORNs to projection neurons (PNs), from ORNs to local interneuron (LNs), and from PNs to the MB Kenyon cells (KCs) are fixed. Lateral inhibition within the AL uses fixed synaptic weights from LNs to PNs. For neuron numbers and their connectivity patterns we here rely on the adult

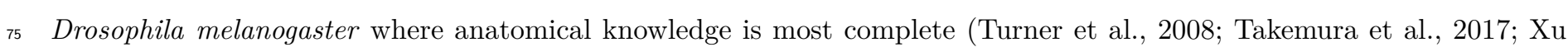
et al., 2020; Aso et al., 2014a). A single MB output neuron (MBON) receives input from all Kenyon cells and plasticity at the synapses between KCs and the MBON enable associative learning (Gütig, 2016; Rapp et al., 2020).

\section{Sparse coding in space and time}

The olfactory system transforms a dense olfactory code in the AL into a sparse stimulus code at the MB level. In the so large population of KCs, a specific odor stimulus is represented by only a small fraction of all KCs (population sparseness) and each stimulus-activated KC responds with only a single or very few action potentials (temporal sparseness).

In our model, temporal sparseness is achieved through the cellular mechanisms of spike-frequency adaptation (SFA, Benda and Herz (2003); Farkhooi et al. (2013); Betkiewicz et al. (2020)) implemented at two levels of the system. ORNs show clear stimulus response adaptation that could be attributed to the spike generating mechanism (Nagel and Wilson, 2011). Based on this experimental evidence we introduced a slow and weak SFA conductance in our model ORNs (see Methods). At the level of the MB, KCs have been shown to express strong SFA-mediating channels (Demmer and Kloppenburg, 2009). This is matched by the SFA parameters of our model KCs (see Methods, (Farkhooi et al., 2013; Betkiewicz et al., 2020)). As an effect of cellular adaptation in ORNs and KCs, odor stimulation (Fig 2 A) results in temporally precise and adaptive responses across all layers of the network (Fig 2 2 B). The effect of SFA implemented

in ORNs is transitive and thus carries over to the postsynaptic PN and LN populations in agreement with experimental observations across species (Stopfer et al., 2003; Wilson et al., 2004; Bhandawat et al., 2007; Krofczik et al., 2009; Watanabe et al., 2012). In the KC population the background firing rate is very low. This is partially due to the outward SFA conductance (Farkhooi et al., 2013) and in agreement with experimental results (Ito et al., 2008). The KC population response is highly transitive where individual responding cells generate only a single or very few response spikes shortly after stimulus onset. This is in good qualitative and quantitative agreement with the temporal sparse KC responses measured in various species (Perez-Orive et al., 2002; Stopfer et al., 2003; Ito et al., 2008; Turner et al., 2008; Gruntman and Turner, 2013; Szyszka et al., 2005; Froese et al., 2014).

Population sparse stimulus encoding at the level of KCs is supported by two major factors. First, the sparse divergentconvergent connectivity between the PNs and the 20 times larger population of KCs is the anatomical basis for sparse

100 odor representation (Huerta et al., 2004; Jortner et al., 2007; Litwin-Kumar et al., 2017; Caron et al., 2013; Betkiewicz et al., 2020). Second, lateral inhibition mediated by the LNs in the AL (Wilson, 2013) facilitates decorrelation of odor representations (Wilson and Laurent, 2005; Schmuker et al., 2011; Wilson, 2013; Campbell et al., 2013) and contributes to population sparseness (Luo et al., 2010; Betkiewicz et al., 2020). The sparse code in the KC population has been shown to reduce the overlap between different odor representations (Luo et al., 2010; Lin et al., 2014; Inada et al., 2017) and 
105 consequently population sparseness is an important property of olfactory learning and plasticity models in insects (Huerta et al., 2004; Huerta and Nowotny, 2009; Wessnitzer et al., 2012; Ardin et al., 2016; Peng and Chittka, 2017; Müller et al., 2018).

The system response to a single odor presentation in Fig. 2B) demonstrates the transformation of a dense olfactory code at the ORN and PN layers into a population sparse representation at the KC layer where less than $<2 \%$ of the total $\mathrm{KC}$ population is active at any time during stimulus presentation. This is in good agreement with quantitative estimates in the fruit fly (Turner et al., 2008; Honegger et al., 2011).

Few-shot learning forms an associative memory of single cues with rewards.

Many insects exhibit a rapid learning dynamics when trained in classical olfactory conditioning tasks. They typically acquire high retention scores (test accuracy $>60 \%$ ) for a binary conditioned response (CR) behavior within only very few trials (e.g. Bitterman et al. (1983); Szyszka et al. (2011); Scheunemann et al. (2013); Pamir et al. (2014)).

Here, we use a classical conditioning paradigm to form associative memories and generate binary CR behavior by training the single MBON in our network (see Fig. 11). We mimic standard experimental lab protocols for classical conditioning (see Fig. 2A). Two different odors are presented as single odor pulses of 500ms duration (see Methods). Multiple independent trials are presented in pseudo-random order where each trial constitutes a single odor stimulus paired with a reward (CS+) or punishment (CS-) shortly after the stimulus presentation.

The system response of the neural network model to a single CS+ stimulus is shown in Fig. 2B. To obtain a neural representation of the odor valence at the MB output (Strube-Bloss et al., 2011; Aso et al., 2014b; Strube-Bloss et al., 2016), the MBON is trained (Gütig, 2016; Rapp et al., 2020) to elicit exactly one action potential in response to a stimulus that is paired with reward (CS+) and zero action potentials when the CS- stimulus is presented (see Methods).

In a first step we quantify the learning performance by considering the accuracy of correctly generated MBON output spikes in response to $\mathrm{CS}+$ and $\mathrm{CS}$ - stimuli after each training trial. The average accuracy over $N=100$ independently trained model instances is shown in Fig. 2C. Learning dynamics of the neural representation of the stimuli show a very steep slope and indicate that memories are formed rapidly with up to $80 \%$ accuracy after presentation of $50(25 \times$ CS+ and $25 \times$ CS-) training trials.

Next, we consider the binary CR behavior depending on whether the MBON generates one or more action potentials in response to a stimulus (positive response) or remains silent (negative response). A conditioned response is correct if the MBON generates a positive response to $\mathrm{CS}+$ cues or a negative response to CS- cues. Results are quantified by a behavioral learning curve (Fig. 2D) representing the median percentage of correctly responding individuals from $N=100$ independently trained models.

In the untrained model, and due to randomly drawn initial synaptic weights, the MBON does not generate any action potentials. Thus all models correctly generate negative behavioral responses to CS- trials from the very beginning and consequently zero correct conditioned responses to CS+ stimuli. After presentation of $3-5$ trials up to $70 \%$ of the trained models are able to generate the correct, appetitive CR to the CS+ stimuli. The learning curve saturates after 10 training trials fluctuating around an asymptotic value of $80 \%$ correct CRs. This reproduces the rapid learning dynamics of insects in classical conditioning experiments and fits qualitatively and quantitatively to the conditioned response behaviors in honeybees (e.g. Bitterman et al. (1983); Pamir et al. (2011); Szyszka et al. (2011); Pamir et al. (2014)).

We conclude that our statically configured sensory network model with a single plastic readout neuron is capable to successfully form associative memories by few-shot learning, replicating the classical conditioning experiments in the typical 

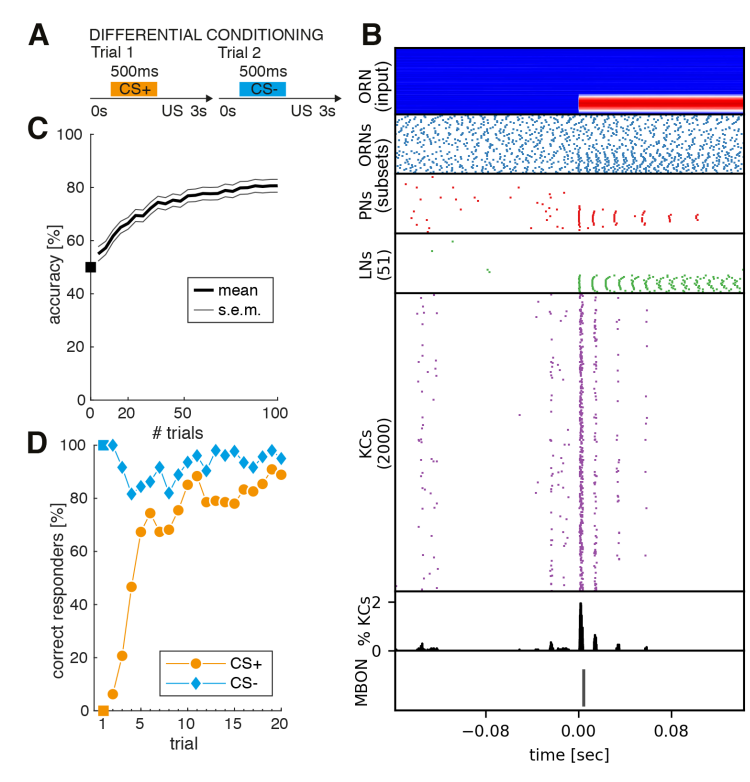

Figure 2: Rapid associative learning with binary behavior response during a classical conditioning task. A: Sketch of a classical conditioning (differential conditioning) paradigm. Two different sensory cues are presented, where one is paired with a reward (CS+), e.g. food, and the other is paired with punishment (CS-). This process is repeated for many trials. After successful learning, the subject is supposed to show a positive binary behavioral response to $\mathrm{CS}+$ cues and no behavioral response to CS- cues. B: System response of the olfactory network model (ORN,AL,MB,MBON) in response to a single $\mathrm{CS}+$ stimulus presented during a classical conditioning paradigm. From top to bottom: Input to the model is provided as noisy current injection into the ORN population. Stimulus onset at $t=0$ sec is clearly visible by magnitude increase of injected current for the sub-population of ORNs that belong to the receptor type sensitive to the presented CS+ odor. Stimulus onset is clearly visible by an increase in spiking activity across all populations of the network. For ORNs and PNs only a relevant subset of 60 and 35 neurons of the total population is shown. ORNs (blue raster plot) sensitive to the presented stimulus show an increase in spiking activity over their spontaneous background activity at stimulus onset which slowly attenuates due to the cellular mechanism of spike frequency adaptation (SFA). A similar attenuating effect is visible in the subset of PNs (red raster plot) that belong to the glomeruli of activated ORNs. This is a transitive effect of SFA implemented in the ORNs, as the PNs do not have SFA implemented. The same can be seen in the LN population (green raster plot). The population of $2000 \mathrm{KCs}$ (magenta raster plot) show temporal and spatial sparse response where during spontaneous background activity (Ito et al., $2008)<1 \%$ of all KCs are active and during stimulus onset only about $2 \%$ of all KCs are active (cf. histogram in black below magenta $\mathrm{KC}$ activity). The transformation of dense code at the ORN population into a temporal and population sparse code at the $\mathrm{KC}$ population have been identified as the two main computational principles of the olfactory system. The last row shows response of the plastic mushroom body output neuron (MBON) after being trained in a classical conditioning paradigm. The MBON correctly responds with a single action potential shortly after CS+ cue onset at $t=0$ sec. C: Learning performance of the readout neuron when pairing a single odor cue with a reward (food). The readout neuron is trained to generate a single action potential in response to odor cues associated with $\mathrm{CS}+$ and remain silent for odor cues associated with CS-. A learning step is performed after each trial if and only if the response of the readout neuron was wrong. The median learning dynamics (red) over $N=100$ independent models show a steep slope up to $80 \%$ accuracy within 50 training trials (approximately $25 \mathrm{CS}+$ and $25 \mathrm{CS}$ - trials). Beyond 50 trials the performance starts to saturate. D: Learning dynamics of a binary behavioral response evoked due to presentation of cues. When the readout neuron fires one or more spikes, it is considered to be a positive response and zero spikes are considered a negative (or no) response. A positive response to a $\mathrm{CS}+$ trial or a negative response to a CStrial is considered to be a correct response. By default the model does not generate any output spike, consequently $100 \%$ of the models correctly respond to CS- trials from the beginning (blue). Behavioral response dynamics to CS+ show a steep slope within 10 trials where $80 \%$ of the models learn to respond correctly. This result closely matches the few-shot learning dynamics observed in insects, e.g. honeybees, on a similar classical conditioning task. 
bioRxiv preprint doi: https://doi.org/10.1101/2020.04.05.026203; this version posted April 5, 2020. The copyright holder for this preprint (which was not certified by peer review) is the author/funder, who has granted bioRxiv a license to display the preprint in perpetuity. It is made available under aCC-BY-NC 4.0 International license.

lab situation. The computational mechanism of population sparseness implemented in our model increases discriminability
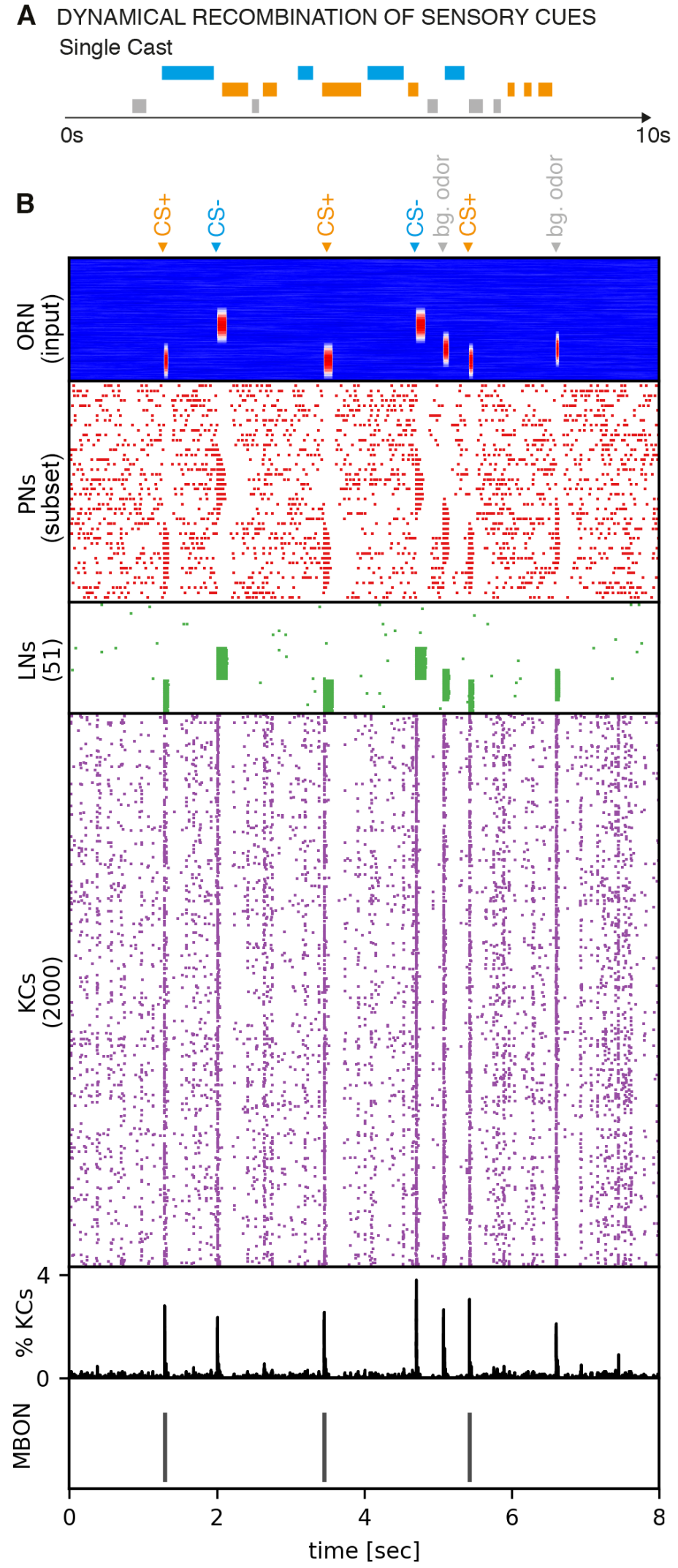

Figure 3: A: Sketch of the problem of dynamic memory recall when experiencing multiple cues over time. In a single trial, multiple cues of different odors are experienced over time. The total number of cues over time varies strongly as well as the number of different odors present (from 2 up to 5 in the data-sets used here). Such a sensory experience can be related to natural conditions, where a flying insect encounters many odor filaments over time, e.g. during "cast" behavior employed for foraging. B System response of the olfactory network model (ORN input, PN, LN, KC, MBON) in response to a dynamical sequence of cues from 3 different odors. Top to bottom: stimulus profiles of model input as current injection into ORNs; different odors activate PNs that belong to different glomeruli, clearly showing the transitive effect of spike-frequency adaptation implemented in ORNs; activity of LNs evoked by different types of odors; response of $\mathrm{KCs}$ with temporal and population sparseness $(\sim 3 \%$ of KCs active during stimulus onset) and spontaneous background activity (Ito et al., 2008); response of the readout MBON with one action potential to each $\mathrm{CS}+$ related cue whereas no action potentials are generated for CS- cues and all other distractor cues. 
Robust dynamic memory recall and background segmentation in complex sensory scenes.

Even though sensory systems are highly specialized sub-systems, in general, each sensory system is in charge of solving several sub-problems. This is particularly true for the olfactory system, which is in charge of odor discrimination (Lin et al., 2014; Riffell et al., 2014), segmentation (Grabska-Barwińska et al., 2016) and possibly other perceptual tasks. Thus, whichever neural representation of external stimuli the system uses, this representation should be general to successfully satisfy all of its intended purposes. We now assess our previously trained model, by associative learning of single odor cues in a classical conditioning paradigm, in a new task of recognizing previously associated odors in complex and dynamic olfactory scenes where several different odor cues are present as sketched in Fig. 3 A. We ask the question how robust and general the learned neural representation is, when many cues of different odor sources appear in complex sequences. If successful, we can conclude that our model is able to use a form of compositionality by means of dynamical memory recall of simpler concepts to solve more complex tasks without explicit re-training. The ability of using previously learned concepts to solve novel tasks, in our case without re-training, is also known as transfer-learning in the scientific field of machine learning. We draw sequences with an average of five non-overlapping cues, each of random duration between $100 \mathrm{~ms}$ and $500 \mathrm{~ms}$ (see Methods). All cues are randomly positioned within $T=10 \mathrm{~s}$ by drawing stimulus onset times from a uniform distribution. Each cue gets randomly assigned to 1 out of up to 5 possible odors. The use of non-overlapping cues follows the rationale presented in Szyszka et al. (2012) who could show that, in nature, filaments originating from different odors do not mix perfectly. The number of possible different odors varies across the 5 different data sets. The data sets are constructed such that the difficulty of odor discrimination increases due to increasing overlap in the ORN receptor activation patterns between odors (similar vs. distinct) and due to an increasing total number of possible odors. Each data-set comprises 200 generated sequences where Fig. 3B shows the system's response to one example sequence with random occurrences of $3 \mathrm{CS}+$ and 4 distractor cues. The ORN receptor activation profile of different odors for all 5 odors is shown in fig. $4 \mathrm{~B}$.

The objective in this task is a neuronal response with a single action potential to each cue occurrence that belongs to the positively associated odor (CS+). For all other cues, CS- as well as any distractor cue, no action potential should be generated. The overall response to a sequence is considered to be correct if and only if the number of action potentials generated by the readout neuron is equal to the number of CS+ cues. Results are shown in fig. AA We find that our previously trained model can successfully generalize to this new task and reaches $\sim 80 \%$ accuracy across all data sets, independent of their complexity. It is thus able to use the previously learned neural representation of single cues and dynamically recalls memories to respond correctly to complex sequences of stimuli of different length. We further find, that our model solves the problem of odor vs. background segmentation (Grabska-Barwińska et al., 2016), by reliably responding to CS+ cues only as quantified by the accuracy of correctly recalled CS+ cues in the sequences.

In a second step we performed the same task, but changed the odor associated with CS + to have large overlap with all 4 distractor odors in terms of ORN receptor activation profile (Fig. $4 \mathrm{D}$ ). We used the same conditioning paradigm with single cues to train the model and test it on the generated sequential data sets (Fig. $4 \mathrm{C}$ ). We find that, despite the large overlap of the receptor profiles of CS+ odor and the distractor odors, our model is still able to generalize reasonably well with accuracy of $50 \%$ correctly detected sequences across all data sets.

We conclude that our model can use a form of compositionality to successfully implement transfer learning without re-training by robustly generalizing memory recall from single cues to complex sequences of multiple cues and distractor cues of variable cue duration. Our results hold for arbitrary distributions of cues in time. As a by-product the model 
A

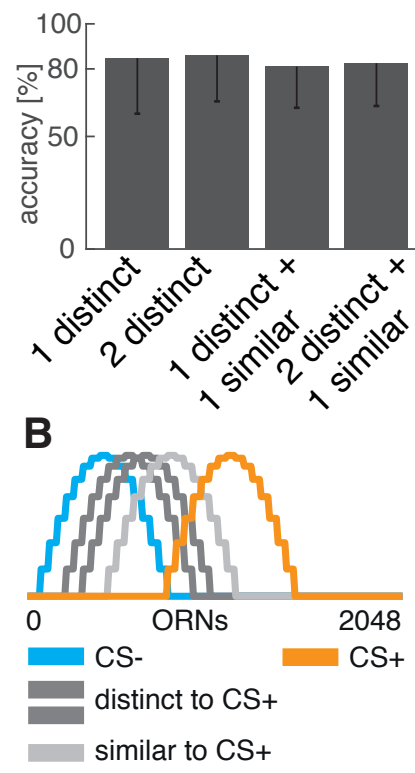

C

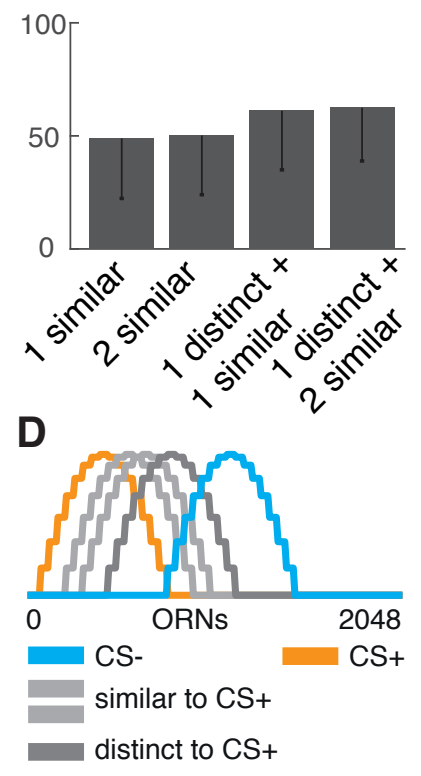

Figure 4: Fast and accurate dynamic memory recall of multiple cues using previously learned associations. A: Results when testing the trained model on the problem of dynamic memory recall of multiple cues. Each of the five different data sets is quantified by accuracy of correctly estimated trials. A single trial constitutes a sequence of multiple sensory cues and is considered correct when the number of CS+ cues within that sequence are correctly predicted by a single action potential per each $\mathrm{CS}+$ cue. Cue distributions are modeled as Poisson-like events. The difficulty increases with each data set in terms of the number of different odors present in a sequence and similarity (ORN stimulus response overlap) of pleasant vs. unpleasant vs. background odors. The results show that the model can successfully solve this new and more complex problem without additional training. B: Similarity of odors in terms of overlap in ORN stimulus response profiles for the 5 different odors. Here, the odor associated with $\mathrm{CS}+$ is distinct from the odor associated with CS- (no overlap). Out of the 3 distractor odors each has a slightly larger overlap with the CS- odor and is considered to be more similar to CS-. C+D: The same type of experiment, but with different ORN response profiles. Now the CS+ odor is very similar to all background odors and renders the problem more difficult. All models have been trained in the same classical conditioning task but using the ORN response profiles shown in panel B,D. Afterwards they are tested on the five generated data sets that contain recombination of multiple cues without retraining. Results show, that the model generalizes reasonably to this problem despite the strong similarity of CS+ and distractor odors. 
solves the problem of odor background segmentation. The results presented in $4 \mathrm{~A}+\mathrm{D}$ suggest, that the functional role of temporal sparseness is to generate precise temporal memory recall up to very short time scales (i.e. for short cue durations and high frequency of cue occurrences).

A
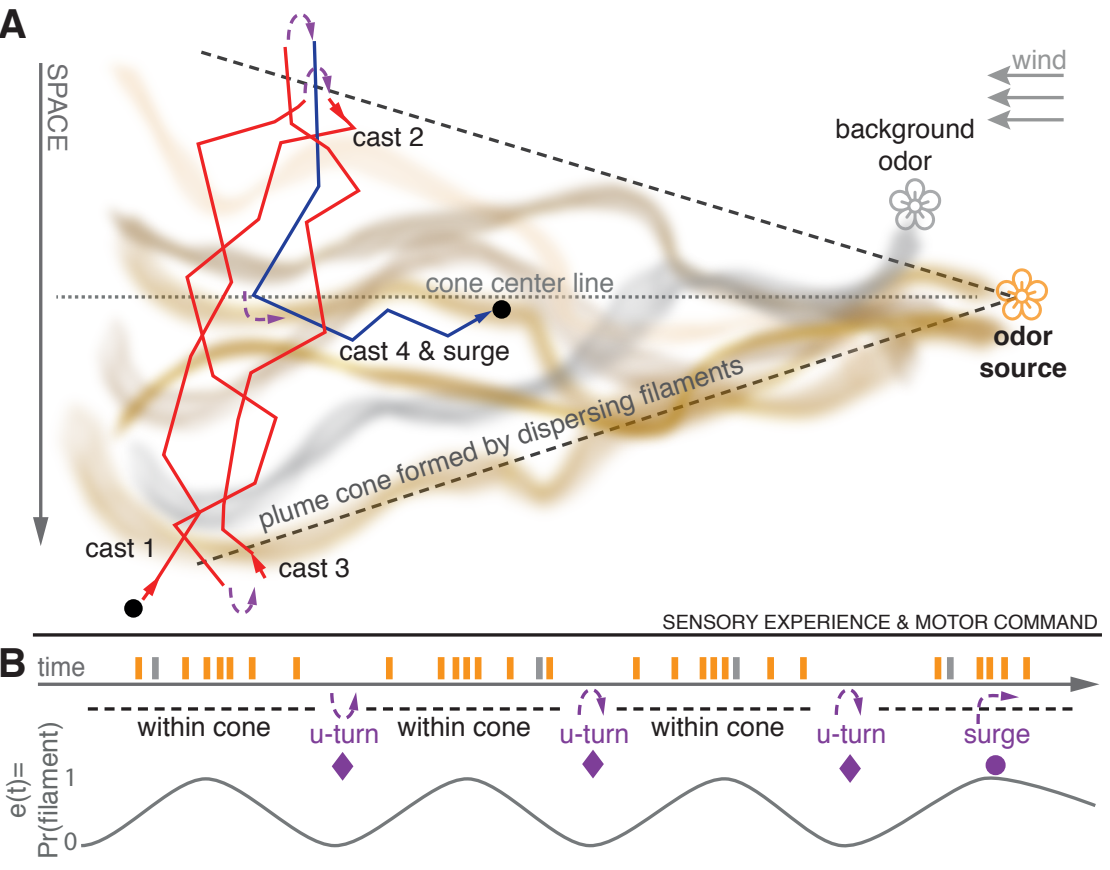

C

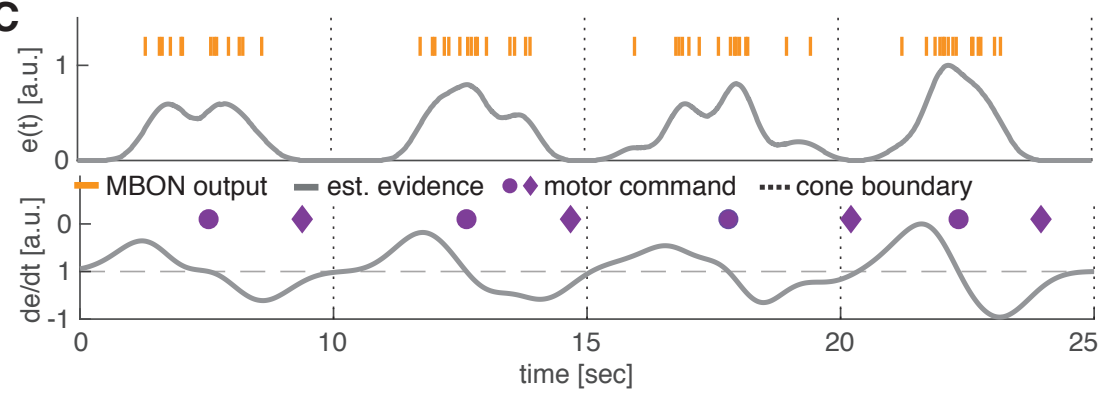

D

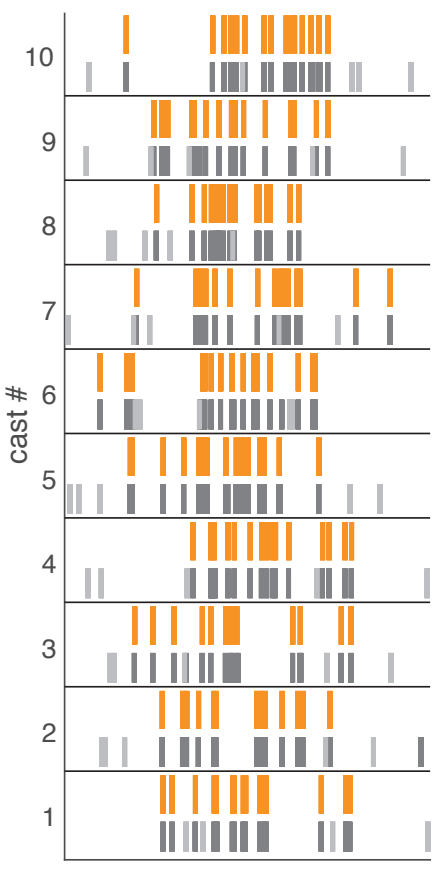

$E$

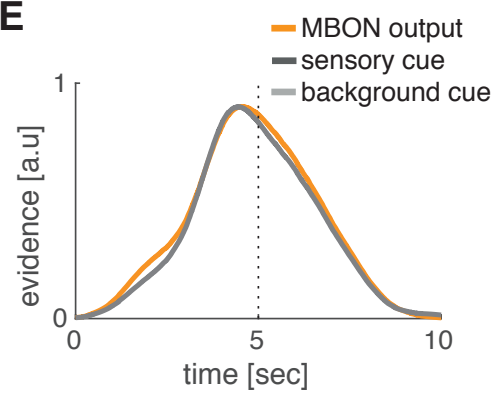

Figure 5: Dynamical sensory processing and motor control serving chemotaxis A: Sketch of a typical olfactory experiment setup in a wind tunnel with a pleasant odor source (orange flower) and a second distractor source (gray flower). Due to turbulence, the odor molecules emitted by a source form dispersing, intermittent filaments within a cone-like boundary that constitutes the odor plume. The plume is modeled as Gaussian distributed filaments. A behaving model insect (here Drosophila melanogaster) performs stereotypic "cast\&surge" behavior to locate the food source. This constitutes alternating between scanning crosswind and U-turning after running past the plume cone boundary where no filaments are present. Eventually after several casts (here 3) it surges upwind until it loses track of the plume cone and starts over. B: Filament encounters during this behavior result in brief on/off, sequential stimuli of the olfactory system. The probability of encountering filaments is $>0$ within the plume and zero outside of the plume. Sensory evidence $e(t)$ can be viewed as a likelihood function of filament encounters, that increases towards the plume's center line and is zero outside of the plume. The properties of this function can be used to generate optimal motor commands for chemotaxis. C: Evidence $e(t)$ and derivative $\frac{d e}{d t}$ over 4 simulated casting trajectories estimated by low-pass filtering the MBON spiking activity. U-turn motor commands are generated when $e(t)$ runs below a fixed threshold $(0.01)$ and surge motor commands are generated when $\frac{d e}{d t}$ turns negative. The motor commands generated by this model closely match the optimal commands as sketched in panel B. D: Spiking activity of the MBON (orange) in response to 10 casting trajectories. The MBON reliably predicts the true sensory cues of simulated filaments (dark gray) and ignores background cues (light gray). E: Smooth PSTH computed over 10 casting trials recovers an accurate estimate of the true underlying sensory cue distribution simulated from a gaussian distribution. 
Sensory evidence accumulation informs motor control for foraging.

We now consider the situation of foraging within a natural environment (fig. 5A). The objective is to locate the food

\section{Distinct functional roles for population and temporal sparse stimulus encoding}

Population sparseness improves discriminability of different stimuli to facilitate associative learning. This has been demonstrated in theory and experiment (Lin et al., 2014; Litwin-Kumar et al., 2017; Caron et al., 2013; Assisi et al., 2020; Nowotny et al., 2003; Jortner et al., 2007). We have shown, that our neural network model implements this feature in a source which emits an attractive odor $(\mathrm{CS}+)$ by utilizing the sensory cues present in its turbulent odor plume. We show that cast \& surge behavior can emerge by accumulation and exploitation of sensory evidence of sequentially experienced individual cues.

For this task, we assume that the occurrence of filaments within a cross-wind slice of the plume are approximately Gaussian distributed. This is a reasonable assumption, particularly in a wind-tunnel setting with laminar flows, as typically used in experimental settings (van Breugel and Dickinson, 2014; Sehdev et al., 2019). However, our model is general and works independent of the actual distribution of filaments. When the insect performs a cast through the plume, it encounters filaments as short-lived discrete, sequential events where each encounter represents a single sensory cue (see sketch in Fig. 5B). Thus, to simulate the sensory experience during casting we generate sequences of cues and distractors, where cue onsets for CS+ odor are drawn from a Gaussian distribution and onsets of distractor cues from a uniform distribution (see Methods). We further assume that the subject has already formed an association of food with the attractive odor, either through learning (e.g. classical conditioning, cf. Fig.2) or through some genetically predetermined innate valence. To this end we again use the trained model from the classical conditioning task above without any further re-training.

We simulate 4 consecutive casting trajectories where the agent senses odor cues of sequentially experienced filament assumes positive values shortly after entering the plume cone and further increases while approaching the plume's center line. When travelling beyond the center line sensory evidence slowly decreases until the agent leaves the plume cone boundary. When sensory evidence drops to zero and after a fix delay, the agent initiates a U-turn motor command to perform another casting trajectory. Responses from our model's readout neuron precisely follow the ground truth of CS+ odor cues as shown by 10 random casting trajectories in Fig. 5D. Performing analysis by averaging of sensory evidence across these 10 casting trajectories yields an average evidence (Fig. $5 \mathrm{E}$ ) that resembles the underlying, true Gaussian profile of the simulated filaments. We thus find that the average estimate produced by our model closely matches the ground truth distribution.

We conclude that the model output provides an accurate and robust estimate of sensory evidence that can be used commands for U-turn and upwind surge behavior, necessary to successfully execute a cast \& surge strategy. Apart from the existence of filaments inside a plume and absence outside a plume's cone, our model does not make any specific assumption regarding the plume's structure and statistics. It thus provides a generic mechanism implemented in a neural system to perform cast \& surge behavior during foraging flights.

\section{Discussion}

biologically realistic way and our results confirm the functional role of population sparseness to support rapid and robust 
memory acquisition through associative learning. Experimental Liu and Davis (2008); Lin et al. (2014) and theoretical (Bennett et al., 2019; Assisi et al., 2020; Litwin-Kumar et al., 2017) studies in the fruit fly strongly suggests that inhibitory feedback through the anterior paired lateral (APL) neuron improves population sparseness in the KC population. The $\mathrm{APL}$ is a GABAergic neuron that broadly innervates the $\mathrm{KC}$ population and likely receives input from KCs in the MB 230 output region. Inhibitory feedback from MB output onto MB input has also been demonstrated in other species (Rybak and Menzel, 1993; Papadopoulou et al., 2011) and blocking of feedback inhibition in the MB reduced population sparseness in the honeybee (Froese et al., 2014). Including an inhibitory feedback loop in our model would further improve robustness of population sparseness and thus not change the our core findings.

Our model demonstrates how temporal sparseness can be exploited to generate short patterned signaling of cue identity. This enables perception of high temporal stimulus dynamics. In our model this is achieved independently of the duration of individual stimulus incidents and their distribution in time and makes temporally precise and robust sensory evidence available. It allows for the ongoing computation of derived estimates such as cue distributions or changes in cue density. Maintaining temporally sparse representations mechanistically supports the principle of compositionality (or Frege principle (Hintikka, 1984)), where an atomic stimulus entity is represented and can be learned by the readout neuron before processing this output. For example by estimation of densities or recombination with other entities to form composite perception or memory read-out. The temporal stimulus dynamics remains intact throughout the system even after learning of stimulus relevance. Thus valence is encoded with the same dynamics and faithfully captures occurrences of relevant cues. This allows compression of code to relevant stimuli while retaining full stimulus dynamics of the external world. Compression of code along the sensory processing pipeline is particularly relevant for small-brained animals like insects, which need to economize on their neuronal resources.

\section{Odor-background segregation: a joint effect of temporal and population sparse cue representation}

The task presented in Fig. 3 implicitly addresses the issue of odor-background segregation. This refers to the problem that in nature cues of multiple odors of different sources are present, either in terms of mixtures or stimulus onset asynchrony due to turbulent conditions (Szyszka et al., 2012; Sehdev and Szyszka, 2019; Grabska-Barwińska et al., 2016; Chakroborty et al., 2016; Deisig et al., 2010; Capurro et al., 2012). For behavior it is relevant to reliably isolate and detect the relevant cues from any background or distractor cues. The results presented in Fig. 4 show that this works nicely in our system. This is achieved by exploiting the joint effect of temporal and population sparseness. Optimal discrimination of cue representation is guarantied by population sparseness and temporal precision by means of temporal sparseness. Our plastic output neuron requires population sparseness for learning and the plasticity rule (Gütig, 2016; Rapp et al., 2020) allows for temporally precise memory recall. We predict that our model can solve the challenge of odor-background segregation.

\section{Rapid learning within few trials}

The ability of insects to quickly form associative memories after 3-5 trials has been demonstrated experimentally (Szyszka et al., 2011; Pamir et al., 2014). In Nowotny et al. (2005) a model has been shown to perform single-shot learning to discriminate odors. However, in general few-shot learning remains a difficult task for computational models (Delahunt and Kutz, 2019; Jiang and Litwin-Kumar, 2019). We find that, when compared with learning dynamics data of real insects (Szyszka et al., 2011; Pamir et al., 2014) our model is able to show realistic learning dynamics that matches 

and it has been shown that MBONs signal odor valence (Hige et al., 2015; Owald et al., 2015; Aso et al., 2014b; Perisse et al., 2016; Strube-Bloss et al., 2016, 2011). We suggest that this valence is then used downstream to execute higher level functions of motor control. At this processing stage it might be integrated with innate valences and other necessary sensory input (Fry et al., 2009; Ache et al., 2019; van Breugel and Dickinson, 2014; Saxena et al., 2018) to form behavioral

\section{Implications for other sensory systems}

Sparse stimulus encoding has been identified as a powerful principle used by higher order brain areas to encode and represent features of the sensory environment in invertebrate (Perez-Orive et al., 2002; Szyszka et al., 2005; Turner et al., 2008; Ito et al., 2008; Honegger et al., 2011) and vertebrate (Hromádka et al., 2008; Vinje, 2000; Wolfe et al., 2010; Isaacson,

Few-shot learning capabilities are also an active area of research in machine learning. Particularly current deep learning methods require massive amounts of training samples to successfully learn a classification model. For example the popular benchmark data sets, ImageNet (Deng et al., 2009) and CIFAR10 (Krizhevsky, 2009), for image classification contain 14 million and 60 million sample images. The Google News dataset used to train language models contains 100 billion words frames from the environment. Clearly, these are numbers a biological organism cannot afford to accumulate. In fact, all animals have been shown to be able to perform few-shot learning which likely is a fundamental skill for survival. We have demonstrated that our neurobiologically motivated approach using spike-based computations is capable to perform few-shot learning with similar speed as real insects to establish an odor-with-reward association. We further showed, that

\section{Innate vs. learned behavior}

Cast \& surge behavior belongs to the innate behavioral repertoire of air-borne insects and emerges from a set of sensorimotor reflexes (van Breugel and Dickinson, 2014). It can be considered as a base strategy wich guarantees survival. The base system can be modulated and improved throughout an animals lifespan by experience-based learning. On the other hand, strategies that emerge solely from learned behavior might require re-training whenever the environment changes. This can be costly in terms of energy, acquisition of samples to learn from or a short life span in general. Here, we assume, that our readout neuron is tuned to a pleasant odor. In the present work this tuning is learned (adaptive process) as we have shown with the classical conditioning task. However, the tuning can generally be learned by other mechanisms, e.g. reinforcement learning. We demonstrated that the existence of such a tuned neuron allows cast \& surge foraging behavior

There are other ways how such a tuned neuron can come about, for example due to genetically predetermined wiring or during development from larval to adult stage. The cast \& surge behavior can be executed on innately valenced olfactory cues and our suggested model for motor control during cast \& surge (Fig. 5 A +B) also works for innate valenced stimuli. Learning is important to adapt behavior to changing environmental circumstances and associative learning provides a decisions.

2010) systems. Sensory systems with similar coding principles may share similar mechanisms when it comes to learning

with the experimental observations. Due to frequent changes in the environment it might be a better strategy to tradeoff fast and reasonable accurate learning against slow and high precision learning. Additionally, acquisition of training 
and multi-modal sensory integration. The mushroom body is a center for integration of multi-modal sensory information. Thus our model can be extended to incorporate multi-modal input from other sensory systems. It is known that olfactory search and foraging strategies do not solely rely on olfactory cues, but require additional sensory information from at least visual cues (Fry et al., 2009; Ache et al., 2019; van Breugel and Dickinson, 2014; Saxena et al., 2018) and wind direction (Wolf and Wehner, 2000; Bhandawat et al., 2010; Álvarez-Salvado et al., 2018; Suver et al., 2019). Extending our model to include additional sensory processing systems for vision and wind direction can provide a comprehensive functional model to study foraging, olfactory search and navigation.

\section{Potential improvement through multiple readout neurons.}

Our current approach only comprises the simplest case of a single readout neuron. This model can be extended to multiple readout neurons. Different readout neurons can be tuned to different odors or groups of odorants. This would allow foraging for different types of food sources and further be useful for multi-modal sensory integration and learning of valences of multiple odors. Another way to use multiple readout neurons is to create an ensemble learning model. Particularly, one can perform bootstrap aggregation (bagging) (Breiman, 1996) to decrease variance of predictions. With this technique, multiple, independent readout neurons are trained for the same target and their outputs are averaged to produce a single output. This approach can be useful when the level of noise increases due to different input models used to drive the network. Another possible extension is to use a single readout neuron to code for multiple odors by associating different number of action potentials to different odors (e.g. 2 or 3). The choice of model for the readout neuron and the plasticity rule allows to do this (Gütig, 2016).

\section{Top-down motor control and lateral horn}

In the current work, generation of motor commands is not computed within the neural network. Integration of sensory evidence is modeled by low-pass filtering of the readout neuron's spike train and its derivative is numerically estimated. In Lundstrom et al. (2008) it has been shown, that a single compartment Hodgkin-Huxley neuron can operate in two computational regimes. One is more sensitive to input variance and acts like a differentiator while in the other regime it acts like an integrator. Similarly Ratté et al. (2015) has shown that the subthreshold current of neurons can encode the integral or derivative of their inputs based on their tuning properties. This could serve as basis for estimating the low-pass filtered sensory evidence and its derivative using neural computations. The turning behavior could be implemented by an always-on neuron serving as a central pattern generator for the motor signal. This neuron can be inhibited by the activity of the readout neuron and only becomes active when no sensory cues are present anymore, which happens shortly after leaving the plume cone. Another option could be to use the cellular mechanism of spike-frequency adaptation to initiate a fast turning movement which slowly decays with a fixed time constant.

\section{Relevance for machine learning and artificial intelligence.}

Learning and building artificial, intelligent agents capable of interacting with their environment are major objectives in the field of machine learning (ML) and artificial intelligence (AI). Deep artificial neural networks (LeCun et al., 2015; Schmidhuber, 2015) have demonstrated great success over the recent years. Particularly, in the domains of image 
becomes evident. They are very specific, single-purpose perceptual systems and poorly generalize to new tasks or changes in an agent's environment (non-stationarities). A few methods to overcome this problem have been proposed, this includes re-training on new tasks and transfer-learning. In the context of deep learning this refers to the method of training a base network on features that are general to all tasks. Afterwards the pre-trained base network is used and the learned features are repurposed to only train a classification layer on the new tasks. However, it turned out that re-training brings up another weakness of deep neural networks, catastrophic forgetting (Kirkpatrick et al., 2017). This term refers to the fact, that after a model has been trained on one task and gets re-trained on a 2nd task, it will completely forget everything it has learned on the previous task. In this work we used a method similarly to the latter approach of transfer-learning but without any additional retraining and we used spike-based learning based on an improved implementation (Rapp et al., 2020) of the Multispike Tempotron (Gütig, 2016). We predict that spike-based methods inspired by biological learning will become increasingly important for artificial intelligence.

\section{Acknowledgments}

This research is supported by the German Research Foundation (grant no. 403329959 to MN) within the Research Unit 'Structure, Plasticity and Behavioral Function of the Drosophila mushroom body' (DFG-FOR 2705, www.unigoettingen.de/en/601524.html).

\section{Author Contributions}

Conceptualization, H.R., MP.N.; Methodology, H.R and MP.N.; Writing - Original Draft, H.R., Writing - Review and Editing, H.R. and MP.N.

\section{Declaration of Interests}

The authors do not declare any conflicts of interest. 


\section{Methods}

\section{LEAD CONTACT AND MATERIALS AVAILABILITY}

This study did not generate new unique reagents.

\section{METHOD DETAILS}

\section{Spiking network model}

All neurons of the olfactory network are modeled as conductance-based leaky integrate-and-fire neurons with spike frequency adaptation (SFA). Specifically, the membrane potential follows the dynamical current balance equation 1. On

For this work the number of neurons within each layer and connectivity schemes are chosen to match the numbers found in the adult Drosophila melanogaster (Takemura et al., 2017; Aso et al., 2014a). Our model comprises 2048 explicitly modeled olfactory recepter neurons (ORNs) organized in 52 different receptor types. ORNs of the same receptor type converge onto the same Glomerulus (52) by feedforward excitatory synapses. Each Glomerulus is formed by a projection neuron (PN) and local interneuron (LN). LNs provide lateral inhibition to all other PNs and LNs. PNs randomly project to a large population (2000) of Kenyon cells (KC) with excitatory synapses such that each KC on average receives input from 6 random PNs. This sparse random convergence implements population sparse responses. The single, plastic mushroom body output neuron is fully connected to all KCs.

We used the cellular mechanism of spike-frequency adaptation (SFA) to achieve temporal sparseness. ORNs are configured to have slow and weak spike-frequency adaptation in accordance with experimental findings (Nagel and Wilson, 2011; Bhandawat et al., 2007; Krofczik et al., 2009). For PNs and LNs SFA has been turned off and KCs are set to produce fast and strong adaptation currents (Wustenberg et al., 2004; Demmer and Kloppenburg, 2009). The property of temporal sparseness can also be achieved by an alternative implementation through feedback inhibition as proposed by Assisi et al. (2020) and Bennett et al. (2019).

The synaptic weights of all connections within the network have been manually determined such that an average background firing rate of $8-10 \mathrm{~Hz}$ is achieved in the LN population. All parameter values used for neurons of each 
bioRxiv preprint doi: https://doi.org/10.1101/2020.04.05.026203; this version posted April 5, 2020. The copyright holder for this preprint (which was not certified by peer review) is the author/funder, who has granted bioRxiv a license to display the preprint in perpetuity. It is made available under aCC-BY-NC 4.0 International license.

population are summarized in tables 1,2, 3, 4. Other parameters used to setup the network (time constants, synaptic weights) are summarized in table 5 . 


$\begin{array}{lll}\text { ORN } & & \\ \text { Parameter } & \text { Value } & \text { Unit } \\ \text { V_threshold } & -57.00000 & \mathrm{mV} \\ \text { b } & 2.00000 & \mathrm{nS} \\ \text { tau_Ia } & 1.00000 & \mathrm{~s} \\ \text { V_rest } & -70.00000 & \mathrm{mV} \\ \text { gL } & 28.95000 & \mathrm{nS} \\ \text { EL } & -70.00000 & \mathrm{mV} \\ \mathrm{C} & 289.50000 & \mathrm{pF}\end{array}$

Table 1: Neuron model parameters used for ORN population.

$\begin{array}{lll}\text { PN } & & \\ \text { Parameter } & \text { Value } & \text { Unit } \\ \text { C } & 289.50000 & \mathrm{pF} \\ \text { tau_Ia } & 1.00000 & \mathrm{~s} \\ \text { V_rest } & -70.00000 & \mathrm{mV} \\ \text { b } & 0.00000 & \mathrm{~S} \\ \text { gL } & 28.95000 & \mathrm{nS} \\ \text { EL } & -70.00000 & \mathrm{mV} \\ \text { v_threshold } & -57.00000 & \mathrm{mV}\end{array}$

Table 2: Neuron model parameters used for PN population.

$\begin{array}{lll}\text { LN } & & \\ \text { Parameter } & \text { Value } & \text { Unit } \\ \text { C } & 289.50000 & \mathrm{pF} \\ \text { tau_Ia } & 1.00000 & \mathrm{~s} \\ \text { V_rest } & -70.00000 & \mathrm{mV} \\ \text { b } & 0.00000 & \mathrm{~S} \\ \text { gL } & 28.95000 & \mathrm{nS} \\ \text { EL } & -70.00000 & \mathrm{mV} \\ \text { V_threshold } & -57.00000 & \mathrm{mV}\end{array}$

Table 3: Neuron model parameters used for LN population. 


$\begin{array}{lll}\text { KC } & & \\ \text { Parameter } & \text { Value } & \text { Unit } \\ \text { C } & 289.50000 & \mathrm{pF} \\ \text { tau_Ia } & 50.00000 & \mathrm{~ms} \\ \text { V_rest } & -70.00000 & \mathrm{mV} \\ \text { b } & 5.00000 & \mathrm{nS} \\ \text { gL } & 28.95000 & \mathrm{nS} \\ \text { EL } & -70.00000 & \mathrm{mV} \\ \text { V_threshold } & -57.00000 & \mathrm{mV}\end{array}$

Table 4: Neuron model parameters used for KC population.

\section{Network}

\begin{tabular}{|c|c|c|c|}
\hline Parameter & Value & Unit & Desciption \\
\hline tau_syn_i & 10.0 & $\mathrm{~ms}$ & time constant inhibit. synapses \\
\hline tau_syn_e & 2.0 & $\mathrm{~ms}$ & time constant excit. synapses \\
\hline tau_ref & 5.0 & $\mathrm{~ms}$ & time constant refractory period \\
\hline w_orn_pn & 1.1282 & $\mathrm{nS}$ & synaptic weight for ORN-PN synapses \\
\hline w_orn_ln & 1.0 & $\mathrm{nS}$ & synaptic weight for ORN-LN synapses \\
\hline $\mathrm{w} \_\ln \_\mathrm{pn}$ & 2.5 & $\mathrm{nS}$ & synaptic weight for LN-PN inhibitory synapses \\
\hline $\mathrm{w} \_\mathrm{pn} \_\mathrm{kc}$ & 14.0 & $\mathrm{nS}$ & synaptic weight for PN-KC synapses \\
\hline
\end{tabular}

Table 5: Other parameters used to configure the model (time constants, synaptic weights, etc.). 
Stimulus response profile of ORNs

The stimulus response profile of ORNs is determined by the ORN tuning curves. We follow a similar method as used in Betkiewicz et al. (2020) where cyclical tuning over receptor types is modeled as half period sine waveforms. Our model comprises $N_{\text {type }}=52$ receptor types and supports 52 different stimuli (e.g. different odors). Where $k_{\text {type }}$ refers to the receptor type index $(\in[0,51])$ and $k_{\text {odor }}$ to the stimulus index $(\in[0,51]) . N_{\text {orn }}=15$ determines the number of receptor types activated by a stimulus. The tuning strength $r$ of the ORNs can be computed as 0.5 cycle of a sine wave with peak amplitude $r_{\max }=1$. In the present work all tuning profiles are normalized to have a peak amplitude of 1 .

$$
\begin{aligned}
& x=\frac{k_{\text {type }}-k_{\text {odor }} \bmod N_{\text {type }}}{N_{\text {orn }}+1} \\
& r=r_{\text {max }} \begin{cases}\sin (x \pi) & \text { for } 0<x<1 \\
0 & \text { else }\end{cases}
\end{aligned}
$$

\section{Model input}

Input to the mushroom body model is modeled as time-dependent, direct current injection into all ORN neurons. In the absence of any stimuli ORNs exhibit spontaneous activity (Nagel and Wilson, 2011). The model input thus consists of spontaneous background activity and stimulus related activity. To generate the background activity, a current time-series is generated for each ORN by simulating shot noise. For each ORN neuron, background activity events are generated from a Poisson process with high rate $(\lambda=300)$ (independent Poisson processes are drawn for each individual neuron). Events of the Poisson process are filtered by a low-pass filter with $\tau=0.6 \mathrm{sec}$. Using this shot-noise model is consistent with experimental findings of odor transduction at the ORNs (Nagel and Wilson, 2011). To induce stimulus related activity to this time-series of ORN $j$ it is multiplied point-wise with a stimulaton protocol time-series $s_{j}(t)$ which is rescaled by a constant determined by the tuning strength $\left(r_{j} \in[0,1]\right)$ to the specific odor of the ORN. This results in a current time series where during stimulus the current magnitude is increased proportional to the ORNs tuning strength and otherwise remains at the magnitude of the background activity.

We define a stimulation protocol function $s(t)$, which is a step function taking on the value 1 at all time points $t$ where a stimulus or sensory cue is active. For each ORN a rescaled instance of the stimulation protocol is defined as $s_{j}(t)=r_{j} s(t)$, where the scaling parameter $r_{j} \in[0,1]$ is given by the stimulus response profile (eq. 6 ) of the ORN to the specific stimulus.

$$
s(t)= \begin{cases}1 & \text { if some stimulus is present } \\ 0 & \text { else }\end{cases}
$$

\section{Sequences of sensory cues}

Each sequence has a duration of 10 seconds. Sequences of sensory cues are generated by drawing the total number of cues within a single sequence from a Poisson distribution with mean $\lambda=8$. Onset times of the cues between 0 and 10 seconds are drawn from a random uniform distribution and it is assured that there is no temporal overlapp between cues. A stimulus relates to a single sensory cue and its duration is drawn uniformly between $[1,200]$ milliseconds. Finally, each sensory cue is associated with a random odor drawn from a fixed set of possible odors (random sampling with replacement and equal probability). This results in sequences with random number of sensory cues, random onset, random duration 
Model of sensory cues within (gaussian) plume

The same procedure is used as above to simulate the experience of sensory cues during a single casting trajectory within a turbulent odor plume. The number of pleasant cues experienced in a casting trajectory is drawn from a Poisson distribution with mean $\lambda=14$. The cue onset times are drawn from a gaussian distribution with $\mu=5, \sigma=1.5$. The number of distractor cues is drawn from a Poisson distribution with mean $\lambda=5$ and are distributed uniformly in time.

Duration of both, pleasant and distractor cues, is drawn uniformly between [100,500] ms. In total 200 different casting trajectories have been generated using this procedure.

\section{Readout Neuron \& Learning rule}

To fit the readout neuron to the stimuli such that it generates 1 spike for pleasant odor stimuli (CS+) and 0 spikes for any other stimuli (CS-) we use a modified implementation of the Multispike Tempotron Gütig (2016); Rapp et al. (2020). Thus, the readout neuron is modeled as voltage-based leaky integrate-and-fire neuron with soft reset following the dynamical equation 7. Incoming spikes evoke exponentially decaying post-synaptic potentials. When the membrane potential reaches the spiking threshold at some time $t_{0}$ an output spike is generated and the membrane potential is reset by the last term of equation 7 .

$$
\begin{aligned}
V(t) & =\underbrace{V_{\text {rest }}}_{:=0}+\sum_{i=1}^{N} \omega_{i} \sum_{t_{i}^{j}<t} \overbrace{K\left(t-t_{i}^{j}\right)}^{\text {exp. PSP kernel }} \\
& -(\underbrace{\vartheta}_{:=1}-V_{\text {rest }}) \sum_{t_{s p}^{j}} e^{-\frac{t-t_{s p}^{j}}{\tau_{m}}}
\end{aligned}
$$

\section{5}

for any other stimuli (CS-). To fit the desired neural code, a training step is performed after each stimulus presentation.

A training step is performed only if the number of spikes generated in response to a stimulus was not correct. The training target is given by the difference between number of output spikes the model generated and the number of output spikes associated with the stimulus. We denote the desired critical threshold value, the voltage value that generates $d=1$ spike, as $\vartheta^{*}$ and the time point where this voltage value is reached by $t^{*}$ (more generally: the critical threshold value to generate $d$ spikes). We briefly sketch the idea and intuition of the Multispike Tempotron learning rule. For detailed derivation of the rule we refer to Gütig (2016) and the section The $\vartheta^{*}$ gradient. The Multispike Tempotron training algorithm works by differentiating the membrane potential of the the critical threshold wrt. to the synaptic weights $(\vec{\omega})$. This can be done since $\vartheta^{*}$ is a regular voltage value, that can be expressed by the neuron's dynamical equation (7), with the special identities shown in equation 10. This allows to take the full derivative as shown in equation 11 .

$$
\begin{aligned}
V_{0}(t) & =\sum_{i=1}^{N} \omega_{i} \sum_{t_{i}^{j}<t} K\left(t-t_{i}^{j}\right) \quad \text { unreset sub-thresh. potential } \\
V(t) & =V_{0}(t)-\vartheta \sum_{t_{s p}^{j}} e^{-\frac{t-t_{s p}^{j}}{\tau_{m}}} \\
\vartheta^{*} & =V\left(t^{*}\right)=V\left(t_{s p}^{j}\right) \quad \text { critical thresh. that makes d spikes } \\
\nabla_{\vec{\omega}} \vartheta^{*} & =\frac{\partial}{\partial \omega} V\left(t^{*}\right)+\sum_{j=1}^{m} \frac{\partial}{\partial t_{s p}^{j}} V\left(t^{*}\right) \frac{\mathrm{d}}{\mathrm{d} \omega} t_{s p}^{j}
\end{aligned}
$$


The gradient of the critical threshold with respect to a single synapse $i$ is given by equation 12 .

$$
\begin{aligned}
& \left(\vartheta_{i}^{*}\right)^{\prime}=\frac{\mathrm{d}}{\mathrm{d} \omega_{i}} \vartheta^{*}=\frac{\mathrm{d}}{\mathrm{d} \omega_{i}} V\left(t^{*}\right)=\frac{\mathrm{d}}{\mathrm{d} \omega_{i}} V\left(t_{s p}^{j}\right) \\
& \left(\vartheta_{i}^{*}\right)^{\prime}=\frac{\partial}{\partial \omega_{i}} V\left(t^{*}\right)+\sum_{j=1}^{m} \frac{\partial}{\partial t_{s p}^{j}} V\left(t^{*}\right) \frac{\mathrm{d}}{\mathrm{d} \omega_{i}} t_{s p}^{j} \quad \text { recursive expr. exists }
\end{aligned}
$$

\section{QUANTIFICATION AND STATISTICAL ANALYSIS}

Spiking network simulations have been performed using BRIAN2 and Python 3.6. Model fitting, data analysis and visualization has been done in MATLAB 2018a and partly in Python using matplotlib.

\section{DATA AND CODEAVAILABILITY}

Code and data sets will be made available through our github profile at: https://github.org/nawrotlab

\section{References}

Ache, J.M., Polsky, J., Alghailani, S., Parekh, R., Breads, P., Peek, M.Y., Bock, D.D., von Reyn, C.R., Card, G.M., 2019. Neural basis for looming size and velocity encoding in the drosophila giant fiber escape pathway. Current Biology

29, 1073 - 1081.e4. URL: http://www.sciencedirect.com/science/article/pii/S0960982219301381, doi:https: //doi.org/10.1016/j.cub.2019.01.079.

Álvarez-Salvado, E., Licata, A.M., Connor, E.G., McHugh, M.K., King, B.M., Stavropoulos, N., Victor, J.D., Crimaldi, J.P., Nagel, K.I., 2018. Elementary sensory-motor transformations underlying olfactory navigation in walking fruit-flies. Elife 7, e37815.

Ardin, P., Peng, F., Mangan, M., Lagogiannis, K., Webb, B., 2016. Using an insect mushroom body circuit to encode route memory in complex natural environments. PLoS computational biology 12, e1004683.

Aso, Y., Hattori, D., Yu, Y., Johnston, R.M., Iyer, N.A., Ngo, T.T., Dionne, H., Abbott, L., Axel, R., Tanimoto, H., et al., 2014a. The neuronal architecture of the mushroom body provides a logic for associative learning. Elife 3, e04577.

Aso, Y., Sitaraman, D., Ichinose, T., Kaun, K.R., Vogt, K., Belliart-Guérin, G., Plaçais, P.Y., Robie, A.A., Yamagata, N., Schnaitmann, C., et al., 2014b. Mushroom body output neurons encode valence and guide memory-based action selection in drosophila. Elife 3, e04580.

Assisi, C., Stopfer, M., Bazhenov, M., 2020. Optimality of sparse olfactory representations is not affected by network plasticity. PLOS Computational Biology 16, e1007461.

Baddeley, B., Philippides, A., Graham, P., de Ibarra, N.H., Collett, T., Husbands, P., 2009. What can be learnt from analysing insect orientation flights using probabilistic slam? Biological Cybernetics 101, 169-182. URL: https: //doi.org/10.1007/s00422-009-0327-4, doi:10.1007/s00422-009-0327-4.

Bahdanau, D., Cho, K., Bengio, Y., 2014. Neural machine translation by jointly learning to align and translate. arXiv: 1409.0473 . 
Baker, K.L., Dickinson, M., Findley, T.M., Gire, D.H., Louis, M., Suver, M.P., Verhagen, J.V., Nagel, K.I., Smear, M.C., 2018. Algorithms for olfactory search across species. Journal of Neuroscience 38, 9383-9389. URL: https://www.jneurosci.org/content/38/44/9383, doi:10.1523/JNEUROSCI.1668-18.2018, arXiv:https://www.jneurosci.org/content/38/44/9383.full.pdf.

Balkovsky, E., Shraiman, B.I., 2002. Olfactory search at high reynolds number. PNAS; Proceedings of the National Academy of Sciences 99, 12589-12593.

Benda, J., Herz, A.V., 2003. A universal model for spike-frequency adaptation. Neural computation 15, 2523-2564.

Bennett, J.E.M., Philippides, A., Nowotny, T., 2019. Learning with reward prediction errors in a model of the drosophila mushroom body. bioRxiv .

Betkiewicz, R., Lindner, B., Nawrot, M.P., 2020. Circuit and cellular mechanisms facilitate the transformation from dense to sparse coding in the insect olfactory system. eNeuro .

Bhandawat, V., Maimon, G., Dickinson, M.H., Wilson, R.I., 2010. Olfactory modulation of flight in drosophila is sensitive, selective and rapid. Journal of Experimental Biology 213, 3625-3635.

Bhandawat, V., Olsen, S.R., Gouwens, N.W., Schlief, M.L., Wilson, R.I., 2007. Sensory processing in the drosophila antennal lobe increases reliability and separability of ensemble odor representations. Nature neuroscience 10, 1474.

Bitterman, M., Menzel, R., Fietz, A., Schäfer, S., 1983. Classical conditioning of proboscis extension in honeybees (apis mellifera). Journal of comparative psychology 97, 107.

Boie, S.D., Connor, E.G., McHugh, M., Nagel, K.I., Ermentrout, G.B., Crimaldi, J.P., Victor, J.D., 2018. Informationtheoretic analysis of realistic odor plumes: What cues are useful for determining location? PLOS Computational Biology 14, e1006275. URL: http://dx.doi.org/10.1371/journal.pcbi.1006275, doi:10.1371/journal.pcbi.1006275.

Breiman, L., 1996. Bagging predictors. Machine Learning 24, 123-140. URL: http://dx.doi.org/10.1007/BF00058655, doi:10.1007/bf00058655.

van Breugel, F., Dickinson, M.H., 2014. Plume-tracking behavior of flying drosophila emerges from a set of distinct sensory-motor reflexes. Current Biology 24, 274-286. URL: https://doi.org/10.1016/j.cub.2013.12.023, doi:10. $1016 / \mathrm{j}$.cub.2013.12.023.

van Breugel, F., Riffell, J., Fairhall, A., Dickinson, M.H., 2015. Mosquitoes use vision to associate odor plumes with thermal targets. Current Biology 25, 2123-2129.

Budick, S.A., Dickinson, M.H., 2006. Free-flight responses of drosophila melanogaster to attractive odors. Journal of experimental biology 209, 3001-3017.

Campbell, R.A., Honegger, K.S., Qin, H., Li, W., Demir, E., Turner, G.C., 2013. Imaging a population code for odor identity in the drosophila mushroom body. Journal of Neuroscience 33, 10568-10581.

Capurro, A., Baroni, F., Olsson, S.B., Kuebler, L.S., Karout, S., Hansson, B.S., Pearce, T.C., $2012 . \quad$ Nonlinear blend coding in the moth antennal lobe emerges from random glomerular networks. Frontiers in neuroengineering 5, 6. URL: http://www.pubmedcentral.nih.gov/articlerender.fcgi?artid=3329896\{\&\}tool= pmcentrez\{\&\}rendertype=abstract, doi:10.3389/fneng. 2012.00006 . 
Cardé, R.T., Willis, M.A., 2008. Navigational strategies used by insects to find distant, wind-borne sources of odor.

Journal of Chemical Ecology 34, 854-866. URL: https://doi.org/10.1007/s10886-008-9484-5, doi:10.1007/ s10886-008-9484-5.

Caron, S.J., Ruta, V., Abbott, L., Axel, R., 2013. Random convergence of olfactory inputs in the drosophila mushroom body. Nature 497, 113.

Celani, A., 2014. Odor landscapes in turbulent environments. Physical Review X 4. doi:10.1103/PhysRevX.4.041015.

Chakroborty, N.K., Menzel, R., Schubert, M., 2016. Environment-specific modulation of odorant representations in the honeybee brain. European Journal of Neuroscience 44, 3080-3093. URL: https://onlinelibrary.wiley.com/doi/abs/10.1111/ejn.13438, doi:10.1111/ejn.13438, arXiv:https://onlinelibrary.wiley.com/doi/pdf/10.1111/ejn.13438.

Connor, E.G., McHugh, M.K., Crimaldi, J.P., 2018. Quantification of airborne odor plumes using planar laser-induced fluorescence. Experiments in Fluids 59, 137.

Crimaldi, J.P., Wiley, M.B., Koseff, J.R., 2002. The relationship between mean and instantaneous structure in turbulent passive scalar plumes. Journal of Turbulence 3, 1-24.

Deisig, N., Giurfa, M., Sandoz, J.C., 2010. Antennal lobe processing increases separability of odor mixture representations in the honeybee. Journal of Neurophysiology 103, 2185-2194. URL: http://jn.physiology.org/cgi/doi/10.1152/ jn.00342.2009\{\%\}5Cnhttp://www.ncbi.nlm.nih.gov/pubmed/20181736, doi:10.1152/jn.00342.2009.

Delahunt, C.B., Kutz, J.N., 2019. Putting a bug in ml: The moth olfactory network learns to read mnist. Neural Networks 118, 54 - 64. URL: http://www.sciencedirect.com/science/article/pii/S0893608019301522, doi:https://doi. org/10.1016/j.neunet.2019.05.012.

Demmer, H., Kloppenburg, P., 2009. Intrinsic membrane properties and inhibitory synaptic input of kenyon cells as mechanisms for sparse coding? Journal of neurophysiology 102, 1538-1550.

Deng, J., Dong, W., Socher, R., Li, L.J., Li, K., Fei-Fei, L., 2009. Imagenet: A large-scale hierarchical image database, in: CVPR09.

Devlin, J., Chang, M.W., Lee, K., Toutanova, K., 2018. Bert: Pre-training of deep bidirectional transformers for language understanding. arXiv:1810.04805.

Egea-Weiss, A., Renner, A., Kleineidam, C.J., Szyszka, P., 2018. High precision of spike timing across olfactory receptor neurons allows rapid odor coding in drosophila. iScience 4, 76-83.

Farkhooi, F., Froese, A., Muller, E., Menzel, R., Nawrot, M.P., 2013. Cellular adaptation facilitates sparse and reliable coding in sensory pathways. PLoS computational biology 9, e1003251.

Froese, A., Szyszka, P., Menzel, R., 2014. Effect of gabaergic inhibition on odorant concentration coding in mushroom 
Fry, S.N., Rohrseitz, N., Straw, A.D., Dickinson, M.H., 2009. Visual control of flight speed in drosophila melanogaster. Journal of Experimental Biology 212, 1120-1130. URL: https://jeb.biologists.org/content/212/8/1120, doi:10. 1242/jeb.020768, arXiv:https://jeb.biologists.org/content/212/8/1120.full.pdf.

Gaudry, Q., Nagel, K.I., Wilson, R.I., 2012. Smelling on the fly: sensory cues and strategies for olfactory navigation

in drosophila. Current Opinion in Neurobiology 22, 216 - 222. URL: http://www.sciencedirect.com/science/ article/pii/S0959438811002285, doi:https://doi.org/10.1016/j.conb.2011.12.010. neuroethology.

Grabska-Barwińska, A., Barthelmé, S., Beck, J., Mainen, Z.F., Pouget, A., Latham, P.E., 2016. A probabilistic approach to demixing odors. Nature Neuroscience 20, $98 \mathrm{EP}$-. URL: https://doi.org/10.1038/nn.4444.

Gruntman, E., Turner, G.C., 2013. Integration of the olfactory code across dendritic claws of single mushroom body neurons. Nature neuroscience 16, 1821.

Gütig, R., 2016. Spiking neurons can discover predictive features by aggregate-label learning. Science 351.

Hein, A.M., Carrara, F., Brumley, D.R., Stocker, R., Levin, S.A., 2016. Natural search algorithms as a bridge between organisms, evolution, and ecology. Proceedings of the National Academy of Sciences 113, 9413-9420. URL: https://www.pnas.org/content/113/34/9413, doi:10.1073/pnas.1606195113, arXiv:https://www.pnas.org/content/113/34/9413.full.pdf.

Hige, T., Aso, Y., Rubin, G.M., Turner, G.C., 2015. Plasticity-driven individualization of olfactory coding in mushroom body output neurons. Nature 526, 258 EP -. URL: http://dx.doi.org/10.1038/nature15396.

Hintikka, J., 1984. A hundred years later: The rise and fall of frege's influence in language theory. Synthese , 27-49.

Honegger, K.S., Campbell, R.a.a., Turner, G.C., 2011. Cellular-resolution population imaging reveals robust sparse coding in the Drosophila mushroom body. The Journal of neuroscience : the official journal of the Society for Neuroscience 31, 11772-85. URL: http://www.pubmedcentral.nih.gov/articlerender.fcgi?artid=3180869\{\&\}tool= pmcentrez $\{\&\}$ rendertype=abstract, doi:10.1523/JNEUROSCI .1099-11.2011.

Hromádka, T., DeWeese, M.R., Zador, A.M., 2008. Sparse representation of sounds in the unanesthetized auditory cortex. PLoS Biology 6, e16. URL: http://dx.doi.org/10.1371/journal.pbio.0060016, doi:10.1371/journal. pbio. 0060016 .

Huerta, R., Nowotny, T., 2009. Fast and robust learning by reinforcement signals: explorations in the insect brain. Neural computation 21, 2123-2151.

Huerta, R., Nowotny, T., García-Sanchez, M., Abarbanel, H.D., Rabinovich, M.I., 2004. Learning classification in the olfactory system of insects. Neural computation 16, 1601-1640.

Inada, K., Tsuchimoto, Y., Kazama, H., 2017. Origins of cell-type-specific olfactory processing in the drosophila mushroom body circuit. Neuron 95, 357-367.e4. URL: http://dx.doi.org/10.1016/j.neuron.2017.06.039, doi:10.1016/j. neuron.2017.06.039.

Isaacson, J.S., 2010. Odor representations in mammalian cortical circuits. Current opinion in neurobiology 20, 328-331. doi:10.1016/j.conb.2010.02.004. 
Liu, X., Davis, R.L., 2008. The gabaergic anterior paired lateral neuron suppresses and is suppressed by olfactory learning. Nature Neuroscience 12, 53-59. URL: http://dx.doi.org/10.1038/nn.2235, doi:10.1038/nn.2235.

Ito, I., Ong, R.C.y., Raman, B., Stopfer, M., 2008. Sparse odor representation and olfactory learning. Nature Neuroscience 11, 1177 EP -. URL: http://dx.doi.org/10.1038/nn.2192.

Jiang, L., Litwin-Kumar, A., 2019. Models of heterogeneous dopamine signaling in an insect learning and memory center. bioRxiv .

Jortner, R.A., Farivar, S.S., Laurent, G., 2007. A simple connectivity scheme for sparse coding in an olfactory system. Journal of Neuroscience 27, 1659-1669.

Kazama, H., Wilson, R.I., 2009. Origins of correlated activity in an olfactory circuit. Nature Neuroscience 12, 1136-1144. URL: http://dx.doi.org/10.1038/nn.2376, doi:10.1038/nn.2376.

Kirkpatrick, J., Pascanu, R., Rabinowitz, N., Veness, J., Desjardins, G., Rusu, A.A., Milan, K., Quan, J., Ramalho, T., Grabska-Barwinska, A., et al., 2017. Overcoming catastrophic forgetting in neural networks. Proceedings of the National Academy of Sciences 114, 3521-3526. URL: http://dx.doi.org/10.1073/pnas.1611835114, doi:10.1073/ pnas. 1611835114 .

Kloppenburg, P., Nawrot, M.P., 2014. Neural coding: Sparse but on time. Current Biology 24, R957-R959. URL: http://dx.doi.org/10.1016/j.cub.2014.08.041, doi:10.1016/j.cub.2014.08.041.

Kree, M., Duplat, J., Villermaux, E., 2013. The mixing of distant sources. Physics of Fluids 25, 091103. URL: https: //doi.org/10.1063/1.4820015, doi:10.1063/1.4820015, arXiv:https://doi.org/10.1063/1.4820015.

Krizhevsky, A., 2009. Learning multiple layers of features from tiny images. Technical Report.

Krizhevsky, A., Sutskever, I., Hinton, G.E., 2012. Imagenet classification with deep convolutional neural networks, in: Pereira, F., Burges, C.J.C., Bottou, L., Weinberger, K.Q. (Eds.), Advances in Neural Information Processing Systems 25. Curran Associates, Inc., pp. 1097-1105. URL: http://papers.nips.cc/paper/ 4824-imagenet-classification-with-deep-convolutional-neural-networks.pdf.

Krofczik, S., Menzel, R., Nawrot, M.P., 2009. Rapid odor processing in the honeybee antennal lobe network. Frontiers in computational neuroscience 2,9 .

LeCun, Y., Bengio, Y., Hinton, G., 2015. Deep learning. Nature 521, 436.

Lillicrap, T.P., Hunt, J.J., Pritzel, A., Heess, N., Erez, T., Tassa, Y., Silver, D., Wierstra, D., 2015. Continuous control with deep reinforcement learning. arXiv:1509.02971.

Lin, A.C., Bygrave, A.M., De Calignon, A., Lee, T., Miesenböck, G., 2014. Sparse, decorrelated odor coding in the mushroom body enhances learned odor discrimination. Nature neuroscience 17, 559 .

Litwin-Kumar, A., Harris, K.D., Axel, R., Sompolinsky, H., Abbott, L., 2017. Optimal degrees of synaptic connectivity. Neuron 93, 1153 - 1164.e7. URL: http://www.sciencedirect.com/science/article/pii/S0896627317300545, doi:https://doi.org/10.1016/j.neuron.2017.01.030. 
Lundstrom, B.N., Hong, S., Higgs, M.H., Fairhall, A.L., 2008. Two computational regimes of a single-compartment neuron separated by a planar boundary in conductance space. Neural Computation 20, 1239-1260. URL: http: //dx.doi.org/10.1162/neco.2007.05-07-536, doi:10.1162/neco.2007.05-07-536.

Luo, S.X., Axel, R., Abbott, L.F., 2010. Generating sparse and selective third-order responses in the olfactory system of the fly. PNAS; Proceedings of the National Academy of Sciences 107, 10713-10718.

Masson, J.B., 2013. Olfactory searches with limited space perception. Proceedings of the National Academy of Sciences 110, 11261-11266. URL: https://www.pnas.org/content/110/28/11261, doi:10.1073/pnas.1221091110, arXiv:https://www.pnas.org/content/110/28/11261.full.pdf.

Mikolov, T., Sutskever, I., Chen, K., Corrado, G.S., Dean, J., 2013. Distributed representations of words and phrases and their compositionality, in: Advances in neural information processing systems, pp. 3111-3119.

Mnih, V., Kavukcuoglu, K., Silver, D., Rusu, A.A., Veness, J., Bellemare, M.G., Graves, A., Riedmiller, M., Fidjeland, A.K., Ostrovski, G., et al., 2015. Human-level control through deep reinforcement learning. Nature 518, 529.

Müller, J., Nawrot, M., Menzel, R., Landgraf, T., 2018. A neural network model for familiarity and context learning during honeybee foraging flights. Biological cybernetics 112, 113-126.

Murlis, J., Elkinton, J.S., Cardé, R.T., 1992. Odor plumes and how insects use them. Annual Review of Entomology 37, 505-532. URL: http://dx.doi.org/10.1146/annurev.en.37.010192.002445, doi:10.1146/annurev.en.37.010192. 002445 .

Murlis, J., Willis, M.A., Cardé, R.T., 2000. Spatial and temporal structures of pheromone plumes in fields and forests. Physiological Entomology 25, 211-222. URL: https://onlinelibrary. wiley.com/doi/abs/10.1046/j.1365-3032.2000.00176.x, doi:10.1046/j.1365-3032.2000.00176.x, arXiv:https://onlinelibrary.wiley.com/doi/pdf/10.1046/j.1365-3032.2000.00176.x.

Nagel, K.I., Wilson, R.I., 2011. Biophysical mechanisms underlying olfactory receptor neuron dynamics. Nature neuroscience 14, 208.

Nowotny, T., Huerta, R., Abarbanel, H.D., Rabinovich, M.I., 2005. Self-organization in the olfactory system: one shot odor recognition in insects. Biological cybernetics 93, 436-446.

Nowotny, T., Rabinovich, M.I., Huerta, R., Abarbanel, H.D., 2003. Decoding temporal information through slow lateral excitation in the olfactory system of insects. Journal of computational neuroscience 15, 271-281.

Owald, D., Felsenberg, J., Talbot, C.B., Das, G., Perisse, E., Huetteroth, W., Waddell, S., 2015. Activity of defined mushroom body output neurons underlies learned olfactory behavior in drosophila. Neuron 86, $417-$ 427. URL: http://www.sciencedirect.com/science/article/pii/S0896627315002214, doi:https://doi.org/10. $1016 / j$.neuron. 2015.03.025.

Pamir, E., Chakroborty, N.K., Stollhoff, N., Gehring, K.B., Antemann, V., Morgenstern, L., Felsenberg, J., Eisenhardt, D., Menzel, R., Nawrot, M.P., 2011. Average group behavior does not represent individual behavior in classical conditioning of the honeybee. Learning \& Memory 18, 733-741. 
Pamir, E., Szyszka, P., Scheiner, R., Nawrot, M.P., 2014. Rapid learning dynamics in individual honeybees during classical conditioning. Frontiers in Behavioral Neuroscience 8. URL: http://dx.doi.org/10.3389/fnbeh.2014.00313, doi:10.3389/fnbeh.2014.00313.

Pang, R., van Breugel, F., Dickinson, M., Riffell, J.A., Fairhall, A., 2018. History dependence in insect flight decisions during odor tracking. PLOS Computational Biology 14, e1005969. URL: http://dx.doi.org/10.1371/journal.pcbi . 1005969, doi:10.1371/journal.pcbi.1005969.

Papadopoulou, M., Cassenaer, S., Nowotny, T., Laurent, G., 2011. Normalization for sparse encoding of odors by a wide-field interneuron. Science 332, 721-725.

Park, I.J., Hein, A.M., Bobkov, Y.V., Reidenbach, M.A., Ache, B.W., Principe, J.C., 2016. Neurally encoding time for olfactory navigation. PLOS Computational Biology 12, e1004682. URL: http://dx.doi.org/10.1371/journal.pcbi. 1004682, doi:10.1371/journal.pcbi.1004682.

Peng, F., Chittka, L., 2017. A simple computational model of the bee mushroom body can explain seemingly complex forms of olfactory learning and memory. Current Biology 27, 224-230.

Pennington, J., Socher, R., Manning, C., 2014. Glove: Global vectors for word representation. Proceedings of the 2014 Conference on Empirical Methods in Natural Language Processing (EMNLP) URL: http://dx.doi.org/10.3115/v1/ D14-1162, doi:10.3115/v1/d14-1162.

Perez-Orive, J., Mazor, O., Turner, G.C., Cassenaer, S., Wilson, R.I., Laurent, G., 2002. Oscillations and sparsening of odor representations in the mushroom body. Science (New York, N.Y.) 297, 359-65. URL: http://www.ncbi.nlm. nih.gov/pubmed/12130775, doi:10.1126/science.1070502.

Perisse, E., Owald, D., Barnstedt, O., Talbot, C.B., Huetteroth, W., Waddell, S., 2016. Aversive learning and appetitive motivation toggle feed-forward inhibition in the drosophila mushroom body. Neuron 90, 1086-1099.

Rapp, H., Nawrot, M.P., Stern, M., 2020. Numerical cognition based on precise counting with a single spiking neuron. iScience, 100852URL: http://dx.doi.org/10.1016/j.isci.2020.100852, doi:10.1016/j.isci.2020.100852.

Ratté, S., Lankarany, M., Rho, Y.A., Patterson, A., Prescott, S.A., 2015. Subthreshold membrane currents confer distinct tuning properties that enable neurons to encode the integral or derivative of their input. Frontiers in Cellular Neuroscience 8. URL: http://dx.doi.org/10.3389/fncel.2014.00452, doi:10.3389/fncel.2014.00452.

Riffell, J.A., Shlizerman, E., Sanders, E., Abrell, L., Medina, B., Hinterwirth, A.J., Kutz, J.N., 2014. Flower discrimination by pollinators in a dynamic chemical environment. Science 344, 1515-1518.

Rybak, J., Menzel, R., 1993. Anatomy of the mushroom bodies in the honey bee brain: the neuronal connections of the alpha-lobe. Journal of Comparative Neurology 334, 444-465.

Saxena, N., Natesan, D., Sane, S.P., 2018. Odor source localization in complex visual environments by fruit flies. Journal of Experimental Biology 221. URL: https://jeb.biologists.org/content/221/2/jeb172023, doi:10.1242/jeb. 172023, arXiv:https://jeb.biologists.org/content/221/2/jeb172023.full.pdf. 
Scheunemann, L., Skroblin, P., Hundsrucker, C., Klussmann, E., Efetova, M., Schwarzel, M., 2013. Akaps act in a twostep mechanism of memory acquisition. Journal of Neuroscience 33, 17422-17428. URL: http://dx.doi.org/10.1523/ JNEUROSCI.1303-13.2013, doi:10.1523/jneurosci.1303-13.2013.

Schmidhuber, J., 2015. Deep learning in neural networks: An overview. Neural Networks 61, 85-117.

Schmuker, M., Yamagata, N., Nawrot, M., Menzel, R., 2011. Parallel representation of stimulus identity and intensity in a dual pathway model inspired by the olfactory system of the honeybee. Frontiers in neuroengineering 4, 17.

Sehdev, A., Mohammed, Y.G., Triphan, T., Szyszka, P., 2019. Olfactory object recognition based on fine-scale stimulus timing in drosophila. iScience 13, 113-124.

Sehdev, A., Szyszka, P., 2019. Segregation of unknown odors from mixtures based on stimulus onset asynchrony in honey bees. Frontiers in Behavioral Neuroscience 13. doi:10.3389/fnbeh.2019.00155. article Number: 155.

Shraiman, B.I., Siggia, E.D., 2000. Scalar turbulence. Nature 405, 639-646. URL: http://dx.doi.org/10.1038/ 35015000, doi:10.1038/35015000.

Simonyan, K., Zisserman, A., 2014. Very deep convolutional networks for large-scale image recognition URL: https: //arxiv.org/abs/1409.1556, arXiv:1409.1556.

Stimberg, M., Brette, R., Goodman, D.F., 2019. Brian 2, an intuitive and efficient neural simulator. eLife 8, e47314. URL: https://doi.org/10.7554/eLife.47314, doi:10.7554/eLife.47314.

Stopfer, M., Jayaraman, V., Laurent, G., 2003. Intensity versus identity coding in an olfactory system. Neuron 39, 991-1004.

Strube-Bloss, M.F., Nawrot, M.P., Menzel, R., 2011. Mushroom body output neurons encode odor-reward associations. Journal of Neuroscience 31, 3129-3140.

Strube-Bloss, M.F., Nawrot, M.P., Menzel, R., 2016. Neural correlates of side-specific odour memory in mushroom body output neurons. Proceedings of the Royal Society B: Biological Sciences 283, 20161270.

Suver, M.P., Matheson, A.M., Sarkar, S., Damiata, M., Schoppik, D., Nagel, K.I., 2019. Encoding of wind direction by central neurons in drosophila. Neuron 102, 828-842.e7. URL: http://dx.doi.org/10.1016/j.neuron.2019.03.012, doi:10.1016/j.neuron.2019.03.012.

Szyszka, P., Demmler, C., Oemisch, M., Sommer, L., Biergans, S., Birnbach, B., Silbering, A.F., Galizia, C.G., 2011. Mind the gap: Olfactory trace conditioning in honeybees. Journal of Neuroscience 31, 7229-7239. URL: http://dx. doi.org/10.1523/JNEUROSCI.6668-10.2011, doi:10.1523/jneurosci.6668-10.2011.

Szyszka, P., Ditzen, M., Galkin, A., Galizia, C.G., Menzel, R., Ditzen, M., Galkin, A., Giovanni, C., 2005. Sparsening and Temporal Sharpening of Olfactory Representations in the Honeybee Mushroom Bodies. Journal of neurophysiology 94, 3303-3313. doi:10.1152/jn.00397.2005.

Szyszka, P., Gerkin, R.C., Galizia, C.G., Smith, B.H., 2014. High-speed odor transduction and pulse tracking by insect olfactory receptor neurons. Proceedings of the National Academy of Sciences 111, 
bioRxiv preprint doi: https://doi.org/10.1101/2020.04.05.026203; this version posted April 5, 2020. The copyright holder for this preprint (which was not certified by peer review) is the author/funder, who has granted bioRxiv a license to display the preprint in perpetuity. It is made available under aCC-BY-NC 4.0 International license.

16925-16930. URL: http://www.pnas.org/content/111/47/16925.abstract, doi:10.1073/pnas.1412051111, arXiv:http://www.pnas.org/content/111/47/16925.full.pdf.

Szyszka, P., Stierle, J.S., Biergans, S., Galizia, C.G., 2012. The speed of smell: odor-object segregation within milliseconds. PloS one 7, e36096. URL: http://www.pubmedcentral.nih.gov/articlerender.fcgi?artid=3338635\{\&\}tool= pmcentrez $\{\&\}$ rendertype=abstract, doi:10.1371/journal . pone. 0036096 .

Takemura, S.y., Aso, Y., Hige, T., Wong, A., Lu, Z., Xu, C.S., Rivlin, P.K., Hess, H., Zhao, T., Parag, T., et al., 2017. A connectome of a learning and memory center in the adult drosophila brain. eLife 6. URL: http://dx.doi.org/10. 7554/eLife.26975, doi:10.7554/elife.26975.

Turner, G.C., Bazhenov, M., Laurent, G., 2008. Olfactory representations by drosophila mushroom body neurons. Journal of neurophysiology 99, 734-746.

Vaswani, A., Shazeer, N., Parmar, N., Uszkoreit, J., Jones, L., Gomez, A.N., Kaiser, L., Polosukhin, I., 2017. Attention is all you need. arXiv:1706.03762.

Vergassola, M., Villermaux, E., Shraiman, B.I., 2007. "infotaxis" as a strategy for searching without gradients. Nature 445, 406-409. URL: http://dx.doi.org/10.1038/nature05464, doi:10.1038/nature05464.

Vickers, N., Christensen, T., Baker, T., Hildebrand, J., 2001. Odour-plume dynamics influence file brain's olfactory code. Nature 410, 466-470. doi:10.1038/35068559.

Vickers, N.J., Baker, T.C., 1994. Reiterative responses to single strands of odor promote sustained upwind flight and odor source location by moths. Proceedings of the National Academy of Sciences 91, 5756-5760.

Vinje, W.E., 2000. Sparse coding and decorrelation in primary visual cortex during natural vision. Science 287, 1273-1276. URL: http://dx.doi.org/10.1126/science.287.5456.1273, doi:10.1126/science.287.5456.1273.

Watanabe, H., Ai, H., Yokohari, F., 2012. Spatio-temporal activity patterns of odor-induced synchronized potentials revealed by voltage-sensitive dye imaging and intracellular recording in the antennal lobe of the cockroach. Frontiers in systems neuroscience 6,55 .

Wessnitzer, J., Young, J.M., Armstrong, J.D., Webb, B., 2012. A model of non-elemental olfactory learning in drosophila. Journal of computational neuroscience 32, 197-212.

Wilson, R.I., 2013. Early olfactory processing in drosophila: mechanisms and principles. Annual review of neuroscience $36,217-241$.

Wilson, R.I., Laurent, G., 2005. Role of gabaergic inhibition in shaping odor-evoked spatiotemporal patterns in the drosophila antennal lobe. Journal of Neuroscience 25, 9069-9079.

Wilson, R.I., Turner, G.C., Laurent, G., 2004. Transformation of olfactory representations in the drosophila antennal lobe. Science 303, 366-370.

Wolf, H., Wehner, R., 2000. Pinpointing food sources: olfactory and anemotactic orientation in desert ants, cataglyphis fortis. Journal of Experimental Biology 203, 857-868. 
Wolfe, J., Houweling, A.R., Brecht, M., 2010. Sparse and powerful cortical spikes. Current opinion in neurobiology 20, 306-312. doi:10.1016/j.conb.2010.03.006.

Wustenberg, D.G., Boytcheva, M., Grunewald, B., Byrne, J.H., Menzel, R., Baxter, D.A., 2004. Current-and voltage-clamp recordings and computer simulations of kenyon cells in the honeybee. Journal of neurophysiology 92, 2589-2603.

Xu, C.S., Januszewski, M., Lu, Z., Takemura, S.y., Hayworth, K.J., Huang, G., Shinomiya, K., Maitin-Shepard, J., Ackerman, D., Berg, S., Blakely, T., Bogovic, J., Clements, J., Dolafi, T., Hubbard, P., Kainmueller, D., Katz, W., Kawase, T., Khairy, K.A., Leavitt, L., Li, P.H., Lindsey, L., Neubarth, N., Olbris, D.J., Otsuna, H., Troutman, E.T., Umayam, L., Zhao, T., Ito, M., Goldammer, J., Wolff, T., Svirskas, R., Schlegel, P., Neace, E.R., Knecht, C.J., Alvarado, C.X., Bailey, D.A., Ballinger, S., Borycz, J.A., Canino, B.S., Cheatham, N., Cook, M., Dreher, M., Duclos, O., Eubanks, B., Fairbanks, K., Finley, S., Forknall, N., Francis, A., Hopkins, G.P., Joyce, E.M., Kim, S., Kirk, N.A., Kovalyak, J., Lauchie, S.A., Lohff, A., Maldonado, C., Manley, E.A., McLin, S., Mooney, C., Ndama, M., Ogundeyi, O., Okeoma, N., Ordish, C., Padilla, N., Patrick, C., Paterson, T., Phillips, E.E., Phillips, E.M., Rampally, N., Ribeiro, C., Robertson, M.K., Rymer, J.T., Ryan, S.M., Sammons, M., Scott, A.K., Scott, A.L., Shinomiya, A., Smith, C., Smith, K., Smith, N.L., Sobeski, M.A., Suleiman, A., Swift, J.,

Takemura, S., Talebi, I., Tarnogorska, D., Tenshaw, E., Tokhi, T., Walsh, J.J., Yang, T., Horne, J.A., Li, F., Parekh, R., Rivlin, P.K., Jayaraman, V., Ito, K., Saalfeld, S., George, R., Meinertzhagen, I., Rubin, G.M., Hess, H.F., Scheffer, L.K., Jain, V., Plaza, S.M., 2020. A connectome of the adult drosophila central brain. bioRxiv URL: https://www.biorxiv.org/content/early/2020/01/21/2020.01.21.911859, doi:10.1101/2020.01. 21.911859, arXiv:https://www.biorxiv.org/content/early/2020/01/21/2020.01.21.911859.full.pdf. 